\title{
Causatividad y estatividad: algunos ejemplos del español ${ }^{1}$
}

\author{
Matías Jaque Hidalgo \\ Universidad de Playa Ancha
}

\begin{abstract}
Resumen
El presente trabajo aborda las características semánticas y sintácticas de los predicados estativos que expresan relaciones causativas. En concreto, centramos nuestro análisis en dos grupos de verbos del español: verbos de alternancia instrumental, del tipo bloquear, tapar u obstruir; y verbos psicológicos de objeto experimentante, del tipo preocupar, molestar o aburrir. Mostraremos que, si bien pueden identificarse en ambos predicados lecturas atélicas y no dinámicas (es decir, estativas), su caracterización como predicados de nivel de estadio (Pylkkänen 2000, Fábregas y Marín 2015) no es descriptivamente suficiente. Propondremos, en cambio, que los estados causativos corresponden siempre a estados davidsonianos
\end{abstract}

1 El presente trabajo se enmarca en el proyecto de investigación "Las variación en las interfaces sintaxis-componentes interpretativos" (FFI2014-56968-C4-3-P), de la Universidad Autónoma de Madrid, integrado en el proyecto coordinado "La variación en las interfaces de la sintaxis con otros componentes del sistema lingüístico" (Universidad Autónoma de Barcelona). Agradezco a Antonio Fábregas, Josefa Martín, Louise McNally, Jaume Mateu, José María Brucart, Olga Fernández Soriano y Juan Carlos Moreno Cabrera por comentarios a versiones previas del trabajo aquí presentado. Todos los errores son, por supuesto, responsabilidad del autor.

2 Para correspondencia, dirigirse a: Matías Jaque Hidalgo (matias.jaque@upla.cl), Universidad de Playa Ancha, Facultad de Humanidades, Departamento de Lingüística; Avenida Playa Ancha 850, Valparaíso, Chile. 
(cf. Maienborn 2005), es decir, estados con un argumento eventivo, que será condición necesaria de una relación causal. Motivaremos esta conclusión desde un modelo de interfaz semántica-sintaxis en la que los argumentos externos marcados como iniciadores no se combinan nunca directamente con estados (puros) (Moreno Cabrera 2003), sino que deben establecer primero una relación temática con un evento (Jaque 2014).

Palabras clave: causación, estatividad, verbos psicológicos, verbos de alternancia instrumental, interfaz sintaxis-semántica.

\title{
Causativity AND STATIVITY: SOME EXAMPLES FROM SPANish
}

\begin{abstract}
This article addresses the syntactic and semantic properties of causative stative predicates. We focus on two Spanish group of verbs: instrumental alternation verbs (bloquear 'to block', obstruir 'to obstruct') and object experiencing psych verbs (preocupar 'to worry', molestar 'to annoy'). We show that, although both groups of predicates exhibit atelic and non dynamic (that is, stative) readings, their aspectual characterization is not properly accounted for by the stage level category (Pylkkänen 2000, Fábregas y Marín 2015). Instead, we claim that causative states are davidsonian states (cf. Maienborn 2005), that is, states with an event argument, which is claimed to be a necessary condition for causative relations. We motivate this conclusion from a model of syntax-semantics interface in which external arguments do not combine directly with (pure) states (Moreno Cabrera 2003), but must establish first a thematic relation with events (Jaque 2014).
\end{abstract}

Keywords: causation, stativity, psych verbs, instrumental alternation verbs, syntax-semantics interface.

Recibido: 06/06/2016 Aceptado: 24/01/2017

\section{INTRODUCCIÓN: ANTECEDENTES CONCEPTUALES}

En términos intuitivos, la noción de causalidad suele estar asociada a la noción de sucesión temporal. De este modo, si A causa B, tendemos a pensar que existe una relación de precedencia entre A y B (cf. Neeleman y van de 
Koot 2012). Un ejemplo prototípico de ello puede hallarse en la relación causal que media entre un disparo y la muerte de una persona. Claramente, el primer evento es anterior a la ocurrencia del segundo. Sin embargo, diversos análisis muestran que, desde un concepto más general de causación, esta relación de precedencia no es en absoluto necesaria; de hecho, para modelar cierto tipo de relaciones causales es incluso equivocada (cf. Talmy 1972). Así, si decimos que una cortina, una puerta o una roca bloquean u obstruyen la salida de una habitación, en al menos una de las lecturas posibles de este predicado se establece una relación, no de sucesión, sino de coextensión temporal entre la permanencia de un objeto en cierta posición y la falta de acceso a la salida. Así, la categoría de los estados causativos, de la que constituye un ejemplo la situación hipotética mencionada, y en la que este trabajo se centra, depende de un concepto de causatividad que la independice del de sucesión temporal.

La asimilación tradicional entre causalidad y sucesión temporal puede deberse a la asociación tradicional que une causación y dinamicidad, entendida como el producto de subeventualidades sucesivas cualitativamente disímiles. Sin embargo, incluso en los análisis semánticos tradicionales, el concepto de CAUSA no añade, por sí mismo, dicha diversidad cualitativa. Considérese, por ejemplo, la relación entre los operadores aspectuales presentada en Dowty (1979), esquematizada en (1):

\section{(1) $[\mathrm{X}$ CAUSE $[\mathrm{BECOME}[\mathrm{P}(\mathrm{Y})]]]$}

En este modelo, CAUSE domina al operador BECOME ('devenir'), que expresa el paso de una entidad desde no tener una propiedad $\mathrm{P}$ (por ejemplo, ser rojo) a tenerla. De acuerdo con Dowty (ibíd.), BECOME se define como el intervalo mínimo en cuyo límite inicial una entidad exhibe la ausencia de una propiedad y en cuyo límite final, su presencia. Esta sucesión temporal de estados diversos queda atribuida, pues, al operador BECOME y no a CAUSE. Queda abierta la posibilidad teórica, así, de que un predicado sea causativo pero no dinámico,siempre que en su estructura semántica CAUSE no domine a un operador BECOME (cf. Pylkkänen 2000, Rothmayr 2009).

La independencia semántica de precedencia temporal y causación se ve asegurada, finalmente, al adoptar un análisis contrafactual de esta última, estrategia seguida por el propio Dowty (1979: 105) y propuesta inicialmente por Lewis (1973). Este puede esclarecerse mediante el siguiente ejemplo:

(2) a. Juan abrió la puerta

b. Si Juan no hubiese actuado de cierta manera y todo lo demás hubiese permanecido igual, entonces la puerta no estaría abierta. 


\section{c. A causa $\mathrm{B}=\mathrm{A} \& \mathrm{~B} \&[\neg \mathrm{A} \rightarrow \neg \mathrm{B}]$}

La relación causal expresada en la oración de (2a) puede parafrasearse, siguiendo el análisis contrafactual de Lewis (1973), mediante el enunciado de (2b). En la versión formal de (2c), se expresa la misma idea: dos proposiciones están causalmente vinculadas si ambas son ciertas y si la negación de la primera implica la negación de la segunda ${ }^{3}$. Lo importante, pues, es que la definición contrafactual de Lewis, como destacan Dowty y, posteriormente, Rothmayr (2009: 43), "does not asume a relation of temporal priority between cause and effect" (Dowty 1979: 109).

Por supuesto, no todos los autores están de acuerdo en que la noción de causación deba independizarse de la de precedencia temporal. Así, Neeleman y van de Koot (2012) reservan para los verbos del tipo bloquear u obstruir la noción de mantenimiento (maintenance), que se distinguiría de la de causación justamente por involucrar coextensión temporal entre causante y causado. Causación y mantenimiento compartirían, no obstante, la noción más general de dependencia (dependence), que correspondería, en lo esencial, al análisis contrafactual de Lewis (1973) y Dowty (1979) arriba apuntado. Por cierto, es perfectamente lícito distinguir un tipo de relación de otra sobre la base de la presencia o ausencia del rasgo de precedencia temporal, pero reservar el término causación exclusivamente para aquella que se encuentra positivamente especificada con respecto a dicho rasgo resulta algo arbitrario, aunque se aduzca para ello que "in our thinking about the world a cause precedes its effect" (Neeleman y van de Koot 2012: 92) . $^{4}$ En este trabajo, nos basaremos en el concepto más general de causación posibilitado por el análisis contrafactual, entendido aquí como el elemento esencial y suficiente.

3 Suele indicarse que un análisis contrafactual de la causación debe complementarse con un criterio de proximidad entre A y B. Así, mientras que tiene sentido decir que el disparo de Juan mató a Pedro, no lo tiene que afirmar que el hecho de que los padres de Juan se conocieran mató a Pedro, aun cuando el análisis contrafactual se aplica en ambos casos. Sin embargo, no está claro que esta restricción sea, en última instancia, de tipo pragmático. Para más discusión, véase Dowty (1979) y Neeleman y van de Koot (2012).

4 De acuerdo con Lewis, establecer una relación de precedencia obligada entre causa y efecto tendría la ventaja de descartar la reversabilidad de la dependencia causal (es decir, que el efecto sea a la vez causa). Sin embargo, este orden de precedencia "rejects a priori certain legitimate physical hyphoteses that posit backward or simultaneous causation" (Lewis 1973b: 566). El análisis contrafactual, en cambio, admite en principio ambos tipos temporalmente contraintuitivos de causación, a la vez que, según argumenta el autor, evita igualmente la reversabilidad de la relación causal. 
En particular, defenderemos la tesis de que los estados que expresan relaciones causativas deben modelarse como eventos no dinámicos temporalmente extensos, es decir, estados davidsonianos ${ }^{5}$. Por otra parte, aquellos predicados que denominaremos estados puros (carentes de argumento eventivo) no pueden expresar causación. De este modo, la presencia de un evento cuenta como un requisito mínimo de la causalidad, que es independiente, así, de la dinamicidad. El artículo se estructura del siguiente modo. En $\S 2$, realizamos un breve repaso de dos propuestas previas sobre los estados causativos, en finés (Pylkkänen 2000) y en español (Fábregas y Marín 2015), en las que se asigna a esta clase de predicados el valor aspectual de estados de nivel de estadio. Posteriormente, en $\S 3$, proporcionamos diagnósticos gramaticales para evaluar el tipo aspectual de los dos grupos de verbos considerados: verbos de alternancia instrumental y verbos psicológicos de objeto experimentante, distanciándonos de las propuestas revisadas en $\S 2$, sobre la base de la distinción empírica entre estados SL y estados davidsonianos. Finalmente, en $\S 4$ se formulan las propiedades semánticas y sintácticas que permitirían modelar las opciones empíricas delimitadas en $\S 3$. El artículo se cierra enumerando las principales conclusiones de esta investigación.

\section{REALIDAD LINGÜÍSTICA DE LOS ESTADOS CAUSATIVOS}

\subsection{Estados causativos en finés: PylkKänen (2000)}

La idea de que no existe una correlación necesaria entre causatividad y dinamicidad ha pasado a ser común en la bibliografía sobre este aspecto, al menos, desde el trabajo de Pylkkänen (2000), quien mostró que los verbos psicológicos de experimentante objeto en finés podían ser causativos y

\footnotetext{
5 Es importante destacar que, aunque tomamos el término de Maienborn (2005), entendemos un estado davidsoniano, fundamentalmente, como un predicado no dinámico con un argumento eventivo, al margen de los criterios descriptivos en que la autora basa su propuesta. Los diagnósticos que empleamos para detectar el argumento eventivo, tal como se verá en la sección 3 y se formaliza en 4 , no coinciden exactamente con los que emplea Maienborn, de manera que la clase definida por la aplicación del concepto puede no ser extensionalmente equivalente. Para más discusión sobre el estatus de las pruebas empleadas en una y otra propuesta, véase Jaque (2014).
} 
estativos a la vez, cuestión que tenía un reflejo directo en su morfología. Así lo muestran los siguientes datos (Pylkkänen 2000: (1), (2)) ${ }^{6}$ :
(3) a. Mikko inhoa-a
hyttysi-ä
Mikko.NOM encontrar.asqueroso-3sg mosquitos-PAR
'A Mikko le desagradan los mosquitos'
b. Hyttyset inho-tta-vat Mikko-a
mosquitos.NOM desagradar-CAUS-3pl Mikko-PAR
'Los mosquitos desagradan a Mikko'
vih-stu-tti-vat
Mikko-a
noticias.NOM enfadar-INC-CAUS.PAS-3pl Mikko-PAR
'Las noticias enfadaron a Mikko'
c. Uutiset

En el ejemplo (3a) tenemos un predicado psicológico (de experimentante sujeto) estativo no causativo. En (3b), se observa la presencia del morfema $t t a$, que codifica causatividad. El carácter morfológicamente independiente de esta información respecto de la dinamicidad se aprecia al advertir que, en el ejemplo (3c), el sufijo causativo tta (en su variante alomórfica para pasado: $t t i)$ coocurre con un sufijo específicamente incoativo (stu).Pylkkänen (2000) mostró, mediante una serie de pruebas gramaticales, que el significado de oraciones como (3b) era, efectivamente, estativo, análogo al de una configuración no causativa de sujeto experimentante como la ejemplificada en (3a). Así, tanto los verbos psicológicos causativos no dinámicos (4a) como los verbos psicológicos no causativos (4b) rechazan la forma progresiva,que es sensible, en finés, a la distinción estado/evento:
(4) a. *Kaisa
on inho- tta- ma- ssa
Matti-a.
Kaisa-NOM es desagradar-CAUS-INF-INESS
Matti-PAR
'Kaisa desagrada a Matti'
b. *Kaisa on inhoa- ma- ssa Matti-a.
Kaisa-NOM es considerar.desagradable-INF-INESS Matti-PAR 'Kaisa considera a Matti desagradable'

\footnotetext{
6 Las convenciones usadas en las glosas son las siguientes: $\mathrm{NOM}=$ caso nominativo, $3 \mathrm{sg} /$ $\mathrm{pl}=$ tercera persona singular/plural, $\mathrm{PAR}=$ caso partitivo, $\mathrm{CAUS}=$ causativo, $\mathrm{INC}=$ incoativo, INESS $=$ inesivo, $\mathrm{PAS}=$ pasado.
} 
La diferencia, no obstante, entre un estado como el de (3a), no causativo, y el (3b), causativo, radica, de acuerdo con la autora, en que el primero corresponde a un predicado IL (individual level) mientras el segundo, a un predicado SL (saje level) ${ }^{7}$. De este modo, solo los estados causativos no dinámicos (5b) admiten cuantificación temporal, no así los verbos psicológicos no causativos (5a), como se observa al emplear el modificador aina 'siempre':

(5) a ?Kerttu aina inhoa- a räntäsadett-a

Kerttu siempreconsidera.desagradable-3SG aguanieve-PAR

'Kerttu siempre considera el aguanieve desagradable'

$\begin{array}{lll}\text { b. Räntäsade inho- tta- a aina } & \text { Kerttu-a } \\ \text { aguanieve desagrada-CAUS-3SG siempre } & \text { Kerttu-PAR } \\ \text { 'El aguanieve siempre desagrada a Kerttu' } & \end{array}$

El carácter episódico de la variante causativa se debería, de acuerdo con el análisis de Pylkkänen, a la presencia de un evento de percepción (física o mental) de un estímulo, cuya vigencia coincide con la vigencia del estado mental denotado por el predicado verbal (cf., para más discusión de esta propuesta, Marín 2015).

\footnotetext{
7 La diferencia entre predicados de individuo (individual level predicates, o IL) y predicados de estadio (stage level predicates, o SL) se remonta, principalmente, a Carlson (1977). Los primeros corresponden a predicados que se aplican directamente sobre individuos, como ser alto, saber inglés o pesar 50 kilos, según la fórmula general $\mathrm{P}(\mathrm{x})$, donde $\mathrm{P}$ es una propiedad y $\mathrm{x}$ un individuo. En cambio, los predicados de estadio se aplican, no sobre individuos, sino sobre "tajadas" espaciotemporales de ellos. Así sucede, por ejemplo, con los predicados locativos (v.g. José está en Santiago), que afectan a una manifestación espaciotemporal de un individuo, pero no a su totalidad, si es que definimos los individuos, justamente, como objetos derivados a partir de una serie estable de manifestaciones espaciotemporales a través de un periodo amplio de tiempo (para Carlson, las entidades ontológicamente básicas son, pues, los estadios y no los individuos). La fórmula correspondiente sería Gs[en.Santiago(s) \& R(s, José)], es decir, existe un estadio s del que se predica su localización en Santiago y que corresponde a una manifestación (expresada por R) del individuo José. En español, se ha propuesto que la distinción IL/SL corresponde a la alternancia copulativa entre ser y estar, análisis defendido, por ejemplo, en Arche (2006). Para más detalles sobre esta distinción, véase Kratzer (1995), Husband (2010), Marín (2013), entre muchos otros.
} 


\subsection{VERBOS PSICOLÓGICOS DE OBJETO EXPERIMENTANTE COMO ESTADOS SL: FÁBREGAS y MARÍN 2015}

Fábregas y Marín (2015) defienden la idea de que los verbos psicológicos de experimentante objeto (en adelante VPEO) conforman estructuras causativas estativas, por oposición a los verbos de experimentante sujeto (VPES: temer, amar, odiar, etc.), que expresarían relaciones estativas más simples. Una consecuencia de ello es que los VPEO designan estados de nivel de estadio (SL), mientras que los VPES designan estados de nivel de individuo (IL). Este patrón, de acuerdo con los autores, se explicaría por la estructura causativa que, en los VPEO, introduce un linde inicial en la estructura eventiva, habilitando su instanciación temporal acotada.

Una prueba empírica de ello es que los VPEO, no así los VPES, admiten cuantificación temporal, como se observa en los ejemplos siguientes (Fábregas y Marín 2015: (25)-(26)):

(6) a. $*\{$ Cuando/siempre que $\}\{$ admira/teme $\}$ tus reacciones, sale de la habitación.

b. ${ }^{*}$ Cada vez que \{odia / prefiere\} las películas de terror, se va al cine.

(7) a. \{Cuando/siempre que $\}$ su hijo \{impresiona/obsesiona $\}$ a María, va al médico.

b. Cada vez que Juan \{asusta/enfada\} a Marta, empieza a llorar.

Según puede observarse, los VPES (temer, odiar, preferir y admirar)rechazan la cuantificación temporal, mientras que los VPEO (impresionar, obsesionar, asustar y enfadar) la admiten sin problemas.

En términos configuracionales, la estructura causativa de un VPEO se formaría sobre la base de un VPES, según se observa en las estructuras siguientes (Fábregas y Marín 2015: adaptado de (3)):

(8) a. VPES:

b. VPEO: $\left[_{\mathrm{SV}}\right.$ Causante $\left[\mathrm{v}_{\text {causa }}\right.$

$$
\begin{aligned}
& {\left[{ }_{\text {SEstado }} \operatorname{Exp}\left[\text { Estado } \mathrm{SD}_{\text {Target }}\right]\right]} \\
& {\left[\text { SEstado }_{\text {Target }} \text { Exp [Estado } \mathrm{SD}_{\text {Tat }}\right]}
\end{aligned}
$$

La configuración de (8a) refleja la estructura sintáctica de un VPES. Al entrar en la sintaxis oracional, el especificador de SEstado -es decir, el experimentante de la proyección estativa que relaciona ambos argumentosse mueve, en una etapa posterior de la derivación sintáctica, al especificador de ST, manifestándose como sujeto. El complemento de Estado, el Target, se realiza, en cambio, como complemento del verbo en la sintaxis explícita. En la estructura de (8b), por otra parte, se añade una estructura causativa 
extra, que abre una nueva posición de especificador a la que puede moverse el $\mathrm{SD}_{\text {Target }}$ (que pasará a interpretarse como causa).

El trabajo comentado posee algunas ventajas interesantes. Como hemos visto, se sostiene que el carácter SL de los VPEO puede derivarse de la presencia de una causa. La causa introduciría un linde inicial que sería suficiente para acotar el predicado temporalmente, lo cual permitiría deducir la distinción SL/IL de una configuración sintáctica independientemente motivada (el valor causal de los VPEO frente al valor estativo simple de los VPES). Con ello, evitaríamos la introducción de distintos tipos de estatividad como primitivos de la teoría. No habría, así, estructuras encargadas de codificar el valor de SL, sino que este se seguiría (como un epifenómeno) de la estructura causal (o por lo menos, de la presencia de un linde inicial que indique que el estado en cuestión es consecuencia de un cambio previo; para más discusión, véase Marín 2013).

Aunque esta opción, al simplificar el repertorio de primitivos aspectuales, es teóricamente atractiva, defenderemos aquí que, sea cual sea la naturaleza específica de los estados SL, esta no puede reducirse a la presencia de una causa, puesto que ello volvería opacas ciertas diferencias empíricas entre algunos estados SL no causativos y los VPEO, predicados que, en cambio, casan de manera natural con otras estructuras eventivas no dinámicas, asimismo no causativas. Revisaremos tales diferencias empíricas en el apartado siguiente, específicamente, en 3.3, luego de aislar las lecturas pertinentes para los grupos de verbos considerados en 3.1 y 3.2 .

\section{NATURALEZA ASPECTUAL DE LOS ESTADOS CAUSATIVOS}

En este apartado abordaremos en detalle la caracterización aspectual de dos grupos de predicados que manifiestan un valor causativo no dinámico (es decir, estativo). Se trata de los verbos llamados de alternancia instrumental (bloquear, tapar, cubrir) y los verbos psicológicos de experimentante objeto (VPEO) (molestar, preocupar, asustar). En particular, mostraremos que tales verbos exhiben, en las lecturas relevantes que aquí nos interesan, un valor atélico no dinámico. Crucialmente, argüiremos que este valor aspectual no puede reducirse a la noción de estado SL. La alternativa que plantearemos es apelar a la categoría de estado davidsoniano (es decir, eventos no dinámicos), que puede y debe distinguirse empíricamente de la de los estados SL. Veamos estos puntos de modo separado. 


\subsection{Delimitación DE LAS LeCturas ESTATIVAS RELEVANTES}

Los verbos del tipo bloquear, tapar y cubrir manifiestan lo que se ha llamado alternancia instrumental (cf. Rothmayr 2009; Jaque 2014, 2016), dado que su sujeto puede corresponder, bien a un Agente, bien a un Instrumento. En la clase que nos ocupa, nos referiremos al sujeto no agentivo como medio, y lo distinguiremos de los instrumentos propiamente tales, por motivos que expondremos más abajo. Considérense los ejemplos siguientes:

(9) a. La policía ${ }_{\mathrm{AG}}$ bloqueó la entrada ${ }_{\mathrm{TEM}}$ con una valla $\mathrm{MED}_{\mathrm{MED}}$.

b. La valla $a_{\mathrm{MED} / \mathrm{CAus}}$ bloquea la entrada $\mathrm{TEM}_{\mathrm{TE}}$.

En (9a), el SP con una valla corresponde al medio con el que se efectúa el evento de bloquear, del que la policía es agente y la entrada, tema. En (9b), en tanto, ese mismo argumento se manifiesta como sujeto, donde se interpreta como causante no agentivo del evento.

La introducción de un medio da lugar a dos variantes aspectuales: en la primera (10a), el objeto en cuestión ocupa gradualmente el espacio (evento dinámico no agentivo); en la segunda (10b), el objeto, como consecuencia de un evento de cambio anterior, permanece en una disposición estática frente al tema, dando lugar a un estado causativo. En esta última opción, se da una relación de coextensión temporal entre la permanencia del objeto y el estado de bloqueo:

(10) a. La valla está bloqueando poco a poco la entrada.

b. La valla está bloqueando la entrada desde hace horas.

Es esta segunda lectura, ejemplificada en (10b), la que nos interesa en este estudio, es decir, aquella donde la duración temporal del predicado no involucra ninguna diversidad cualitativa entre sus distintos subintervalos. Se trata, pues, de situaciones homogéneas o no dinámicas. Otros ejemplos pertinentes se ofrecen en (11):

(11) a. La cortina tapa la luz.

b. Los árboles cubren el resplandor del lago.

c. Ese clavo traba el cajón.

d. El colesterol obstruye las arterias.

Desde un punto de vista conceptual, los sujetos de (11) (así como los de (9b) y (10)) se conciben como entidades cuya presencia impide la realización de 
un curso de sucesos alternativo. Así, si la cortina tapa la luz, la luz no puede ingresar libremente; si el colesterol obstruye las arterias, la sangre no puede fluir; si la valla bloquea la entrada, una persona no puede hacer uso de ella, etc. En otras palabras, es viable pensar en un análisis contrafactual(causativo) de las situaciones denotadas por estas oraciones.

Por otra parte, según hemos adelantado, deben distinguirse estos sujetos de aquellos SSDD que poseen genuinamente el papel temático de instrumento. Considérense los verbos abrir o sanar (cf. Moreno Cabrera 2003: 281-294), que manifiestan una alternancia o diátesis instrumental análoga a la de bloquear ejemplificada en (9):

(12) a. Juan abrió la puerta con esta llave.

a'. Esta llave abrió la puerta.

b. El médico sanó la herida con esta pomada.

b'. Esta pomada sanó la herida.

Sin embargo, existen diferencias importantes entre ambos casos. Una característica de los SSDD destacados en (12a-b) es que corresponden a entidades cuya participación se restringe a la subfase inicial de la eventualidad denotada, sin que sea en absoluto necesario que intervengan en el estado resultante de dicha eventualidad. Así, la llave interviene en la acción que desencadena la apertura de la puerta, pero no durante el estado en que esta apertura se mantiene vigente. Una consecuencia de ello es que la eventualidad en su conjunto es necesariamente dinámica, toda vez que incluirá como mínimo dos fases cualitativamente disímiles: una en que interviene un instrumento (se mencione o no un agente) y otra en que se asocia una entidad con una propiedad (el estado resultante). La disociación del instrumento con el estado resultante permite predecir que la variante en que el SD en cuestión es sujeto no corresponderá aspectualmente a un estado léxico. Así, en contextos donde, a diferencia de lo que sucede con el empleo del indefinido en (12a'-b'), se favorece sintácticamente una lectura de estado, esta será genérica y no episódica. Comparemos las siguientes oraciones:

(13) a. Esta publicidad \{bloquea/tapa/obstruye $\}$ el paisaje.

b. Esta llave abre la puerta de salida.

c. Esta pastilla sana el dolor de cabeza.

Una diferencia crucial entre (13a) y (13b-c) es que en aquella el presente posee un valor episódico: se describe un estado de cosas válido en el momento de habla (la publicidad ocupa, de hecho, un lugar). En (13b-c), en 
cambio, el presente posee un valor genérico-disposicional ${ }^{8}$ : no se describe ninguna situación en la que, de hecho, la llave esté siendo introducida en la puerta ni en la que la pastilla esté sanando un dolor; se indica únicamente que, sin que sea necesario que hayan sido empleadas alguna vez para ello, dichas entidades tienen la capacidad de abrir esa puerta o de sanar ese dolor. Siguiendo a Carlson (2012), podemos entender la genericidad como una operación que se aplica sobre un evento más básico; así, (13b-c) denotarían un estado derivado o de aspecto externo (cf. Verkuyl 1993) y no, en rigor, una situación no dinámica9 .

De modo general, diremos que los argumentos que expresen papeles temáticos de una eventualidad estativa deben satisfacer lo que llamaremos condición de coextensión (Jaque 2014), según la cual la intervención de la entidad denotada por el argumento en cuestión debe abarcar como mínimo el tiempo en el que es válido el estado involucrado. Si un argumento restringe su participación a una subfase inicial, como es el caso de los instrumentos, introducirá heterogeneidad y, con ello, dinamicidad, en la situación denotada. En cambio, los medios son coextensivos con el estado resultante y dan lugar, en el cambio diatético comentado, a eventualidades no dinámicas sin necesidad de recibir una lectura genérico-disposicional ${ }^{10}$.

8 Descártese la lectura, altamente restringida en español, en la que un predicado dinámico puede ser empleado en presente con valor episódico ("presente de reportaje"), típica de, por ejemplo, locutores deportivos (v.gr. Alexis se adelanta, dispara y es gol). Nótese que, si se adopta esta lectura marcada, el valor aspectual del predicado sigue siendo dinámico, de modo que seguiría siendo válida la restricción que impide a los instrumentos dar lugar a predicados estativos.

9 La diferencia comentada entre medios e instrumentos puede apreciarse en otros contrastes ajenos a la diátesis en que uno de estos argumentos ocupa la posición de sujeto. Así, colgar un cuadro con un martillo (donde tenemos un instrumento) se distingue de colgar un cuadro con un clavo (donde tenemos un medio) en que solo el clavo debe permanecer durante la vigencia del estado en el que el cuadro cuelga. Como consecuencia de esto, solo el segundo SP puede modificar a la construcción resultativa correspondiente: El cuadro está colgado\{́con un clavo/??con un martillo\}. La segunda opción es pragmáticamente poco aceptable, ya que nos obliga a imaginar que el martillo funciona, no como el instrumento que se empleó para fijar el cuadro en el muro, sino como su soporte. De este modo, los instrumentos se parecen a los agentes en que no se les exige coextensión temporal con el estado resultante.

10 La condición de coextensión puede entenderse como necesaria pero no suficiente para la configuración de la estatividad. Así, es posible encontrar ejemplos de eventos dinámicos atélicos que tomen instrumentos coextensivos sin que, por ello, obtengamos estatividad: v.gr. Pedro martilleó el clavo con una piedra durante una hora. De todos modos, incluso en estos casos puede pensarse, más que en coextensión estricta, en una serie de eventos en que interviene un instrumento que es, en conjunto, coextensiva con un evento atélico mayor. 
Por otra parte, los verbos de experimentante objeto presentan lecturas análogas a las que hemos despejado más arriba para verbos del tipo bloquear. Como es bien sabido (Belleti y Rizzi 1988, Pesetsky 1995, Landau 2009, Marín y McNally 2011), los verbos psicológicos se manifiestan en distintas configuraciones sintácticas, que han suscitado una nutrida discusión sobre la exacta naturaleza aspectual y argumental que en ellas interviene. Así, algunas de las principales son:

(14) a. Napoleón ${ }_{\mathrm{EXP}}$ temía a las arañas ${ }_{\text {темА }}$.

b. Napoleón ${ }_{\text {CAUSA }}$ preocupaba a los líderes europeos ${ }_{\mathrm{EXP}}$.

c. A los líderes europeos ${ }_{\mathrm{EXP}}$ les preocupaba Napoleón ${ }_{\mathrm{TEMA}}$.

d. Los líderes europeos ${ }_{\mathrm{EXP}}$ se preocuparon.

Sobre la primera alternativa (14a), en que el experimentante es sujeto y el tema objeto, existe cierto consenso sobre su naturaleza aspectual estativa (Landau 2009, Marín 2011, Marín 2015). Mayor debate han generado las configuraciones de (14b-d) ${ }^{11}$. La postura tradicional (Belleti y Rizzi 1988, Pesetsky 1995, Grimshaw 1990, Tenny 1994, Moreno Cabrera 2003, cf. Cifuentes Honrubia 2015) atribuye un valor eventivo-dinámico a las variantes (14b), con objeto experimentante acusativo y sujeto causa, y (14d), con verbo reflexivo y sujeto experimentante, frente al carácter estativo de (14c), con objeto experimentante dativo y tema nominativo. Así, existiría una correlación entre la asignación de caso acusativo/dativo y un valor aspectual eventivo/estativo, respectivamente (Strozer 1976, Arad 1999, Marín 2015).

11 Una de las principales fuentes de debate sobre ejemplos como los de (14b), frente a (14a), es que suponen un aparente cruce diatético de papeles temáticos y posiciones sintácticas, de suerte tal que, en la configuración de (14a), el tema es objeto; mientras en (14b), sujeto. Esta posibilidad es conflictiva bajo la Hipótesis de la Uniformidad de Asignación de Papeles Temáticos (UTAH, por sus siglas en inglés, Baker 1988), según la cual los papeles temáticos se asignan en posiciones sintácticas siempre iguales, a menos que exista un cambio diatético (en el marco teórico respectivo, una transformación que cambie la distribución argumental fijada en la Estructura Profunda). Las principales soluciones a este problema han sido la inacusativa (Belleti y Rizzi 1988), según la cual los verbos de experimentante objeto corresponden en verdad a construcciones inacusativas (es decir, donde el argumento en posición de sujeto provendría de una posición de objeto marcado como tema en la estructura profunda, conservando la uniformidad diatética entre ambas clases de verbos); y la solución temática (Pesetsky 1995), que consiste en asignar al sujeto de construcciones como las de (14b) (aunque no así al dativo de (14c)) el papel temático de Causa, no de Tema. De este modo, no existiría realmente una infracción a la UTAH, en la medida en que, asumiendo una jerarquía de prominencia argumental Causa $<\operatorname{Exp}<$ Tema, es esperable que la realización sintáctica de verbos como temer difiera de la de verbos como preocupar. Para más discusión, véase Marín (2015). 
Actualmente, ha ganado terreno la idea según la cual, como mencionábamos en el apartado 1, la variante con experimentante objeto acusativo es igualmente estativa (Rothmayr 2009, Marín 2011, 2013, 2015), aunque se distingue de (14c) por el carácter causativo de su sujeto. Seguiremos aquí esta línea, centrándonos en las construcciones del tipo (14b), para las cuales aduciremos en los apartados siguientes pruebas aspectuales que confirman su carácter no dinámico.

Nótese, por lo pronto, que, de ser así, existe un paralelismo aspectual y argumental entre los verbos del tipo bloquear y los del tipo preocupar(lo). En ambos casos, asistimos a una relación de causación no dinámica.

\subsection{Atelicidad y falta De DinAmicidad}

En términos aspectuales, mostraremos aquí que en ambas construcciones nos vemos frente a predicados atélicos y no dinámicos, siguiendo, en lo general, la caracterización de Rothmayr (2009) para los verbos de alternancia instrumental y de Marín (2011),Rothmayr (2009) y, en especial, Fábregas y Marín (2015) para los verbos de experimentante objeto (VPEO) ${ }^{12}$.

Con respecto a la telicidad, tanto los verbos del tipo bloquear como los VPEO rechazan modificadores adverbiales del tipo en x tiempo (cf. Fábregas y Marín 2015: 179):

(15) a. \#La valla bloqueó la entrada en dos horas.

b. \#Las cortinas taparon la pantalla en dos horas.

c. ??Los rumores molestaron al jefe en cinco minutos.

d. ??La corrupción preocupó a la gente en dos semanas.

(16) a. La tormenta hundió el barco en dos minutos.

b. \#Los niños rieron a carcajadas en cinco minutos.

Como puede observarse, la introducción del modificador en dos horas, tanto con bloquear (15a) como con tapar (15b) desencadena una lectura causativo-

12 Para el análisis de los VPEO nos restringiremos, por claridad de la exposición, a construcciones con un sujeto causa y excluiremos sujetos agentes volitivos. De acuerdo con Marín (2011), aun en estos casos, como sucede con verbos como molestar, es posible sostener una lectura de estado (atélica y no dinámica). Verbos como preocupar, de acuerdo con el mismo autor, serían no agentivos (es decir, en Juan preocupa a María, Juan actúa como causa, no como agente voluntario). Por último, solo unos pocos verbos psicológicos se comportarían como verbos genuinamente dinámicos, entre los que contarían humillar y seducir. 
dinámica, es decir, en la que se produce un cambio de estado inducido por una entidad externa (la valla, las cortinas), si bien, en este caso, carece de control volitivo sobre el estado de cosas resultante. Un resultado similar se observa en el caso de los VPEO (15c-d). Los ejemplos de (16) muestran la conducta de verbos ajenos a estas clases con estos mismos modificadores: así, un verbo causativo télico como hundir es perfectamente compatible con en dos minutos, mientras que el mismo SP, con un verbo inergativo (dinámico atélico) como reír, mide el tiempo precedente al comienzo del evento ('tardaron cinco minutos en comenzar a reír') pero en ningún caso la duración efectiva de la risa misma.

Un segundo contexto que apunta en la misma dirección es la distribución de las construcciones absolutas de participio introducidas por la fórmula una vez (cf. Marín 2011, 2015; Fábregas y Marín 2015):

(17) a. \#Una vez bloqueada la salida, la gente se alarmó.

b. \#Una vez tapada la pantalla, comenzaron las pifias del público.

c. *Una vez molestado el jefe, reinó el silencio en la oficina.

d. *Una vez preocupada tu madre, la tendrás encima todo el día.

(18) a. Una vez hundido el barco, los soldados descorcharon un champán.

b. *Una vez reídos los niños, el payaso se sintió satisfecho.

Como puede apreciarse en los ejemplos (17a-b), los verbos de alternancia instrumental dan lugar, en este contexto, a una lectura dinámica (agentiva o no), desactivando la lectura en que el medio es coextensivo con el estado resultante. En el caso de (17c-d), se aprecia una fuerte inaceptabilidad de la construcción de participio por parte de los VPEO. Nuevamente, el contraste con verbos ajenos a estas clases resulta ilustrativo. Un verbo télico como hundir admite la construcción absoluta sin problemas, mientras que un verbo inergativo como reír la rechaza de modo categórico.

Veamos ahora algunos contextos relacionados con la detección de dinamicidad. El primer criterio que emplearemos es la admisibilidad del adverbio lentamente, rechazado por predicados no dinámicos (cf. Jaque 2014, 2016; cf. Cinque 1999, Marín 2011, Fábregas y Marín 2015: 180):

(19) a. \#La roca bloquea lentamente el camino.

b. \#La cortina tapa lentamente la única ventana disponible.

c. ??Los rumores molestan lentamente al jefe.

d. ??La corrupción preocupa lentamente a la ciudadanía. 
(20) a. El barco se hunde lentamente.

b. Los niños ríen lentamente.

En el caso de verbos del tipo bloquear (19a-b), el adverbio lentamente selecciona una lectura dinámica no agentiva (puesto que el sujeto refiere una entidad no volitiva). En tanto, la aceptabilidad de los VPEO (19c-d) es baja, indicio de que no hay cambio interno. Finalmente, tanto hundir como reír se comportan como predicados dinámicos, mostrando que este criterio es genuinamente independiente de la telicidad.

Por último, emplearemos la construcción parar de+ inf, que selecciona exclusivamente predicados dinámicos, frente a dejar de + inf, que se aplica indistintamente a estados y eventos (cf. de Miguel 1999, Marín 2011, Fábregas y Marín 2015: 180):

(21) a. El agua \{paró de/dejó de\} bloquear la cañería.

b. El colesterol \{paró de/dejó de\} tapar la arteria.

c. Los rumores \{??pararon de/dejaron de\} molestar al jefe.

d. La corrupción \{??paró de/dejó de\} preocupar a la ciudadanía.

(22) a. El barco \{paró de/dejó de\} hundirse.

b. Ya \{paró de/dejó de\} llover.

c. Obama \{*paró de/dejó de\} habitar la Casa Blanca.

En los ejemplos de (21a-b), la selección de parar / dejar se correlaciona con una lectura dinámica o indistinta entre estado y evento, respectivamente ${ }^{13}$. En el caso de (21c-d), existe una fuerte preferencia por la opción dejar de + inf. Finalmente, los ejemplos de (22) muestran la distribución de estas perífrasis con verbos de otras clases. Así, un verbo dinámico télico admite ambos auxiliares (22a), tal como sucede en el caso de un verbo dinámico atélico como llover (22b). (22c), por otra parte, muestra que un verbo estativo admite igualmente dejar de; es decir, dicha perífrasis marca el

13 Un revisor anónimo nos hace notar que la aceptabilidad de (21a-b) con parar de es baja. Es posible que, en este caso, el empleo de bloquear mejore en una lectura dinámico-agentiva, como en el siguiente ejemplo: "Elección tras elección, con demagogia y propaganda, no pararon de bloquear todos los gobiernos de Catalunya desde el primer día [...]" (politicaxxi. net, [recuperado: 20.01.17]). En este caso, seguiría siendo válido que la selección de parar es incompatible con la lectura estativa o no dinámica, aunque impondría restricciones más severas sobre el papel temático del sujeto. De todos modos, si se observan los ejemplos (22ab), donde tenemos un sujeto paciente y un inergativo impersonal, puede concluirse que la distribución entre parar y dejar depende más de la dinamicidad que de la agentividad. 
término de la extensión temporal de una eventualidad, sea esta dinámica o estativa, mientras que parar de se aplica exclusivamente a eventualidades dinámicas. Nótese, finalmente, que la distribución de parar de no depende de la agentividad del predicado, sino genuinamente de su dinamicidad, como atestiguan los ejemplos (22a-b), anticausativo e inergativo impersonal, respectivamente.

Los resultados de los contextos aplicados anteriormente se sintetizan en la siguiente tabla:

\begin{tabular}{|l|l|c|c|c|c|}
\hline $\begin{array}{l}\text { Propiedad } \\
\text { semántica }\end{array}$ & Contexto & $\begin{array}{c}\text { Alternancia } \\
\text { instrumental } \\
\text { (bloquear, } \\
\text { lectura no } \\
\text { dinámica) }\end{array}$ & $\begin{array}{c}\text { VPEO } \\
\text { (preocupar) }\end{array}$ & $\begin{array}{c}\text { Verbos } \\
\text { dinámicos } \\
\text { télicos } \\
\text { (hundir) }\end{array}$ & $\begin{array}{c}\text { Verbos } \\
\text { dinámicos } \\
\text { atélicos } \\
\text { (reir) }\end{array}$ \\
\hline \multirow{2}{*}{ Telicidad } & \begin{tabular}{l} 
Enx tiempo \\
\cline { 2 - 6 } \\
pna vez +
\end{tabular} & - & - & + & - \\
\hline participio & - & - & + & - \\
\hline
\end{tabular}

Tabla 1. Conducta de los estados causativos como predicados atélicos y no dinámicos

Podemos concluir, pues, en línea con los resultados de Rothamayr (2009), para el caso de los verbos de alternancia instrumental del alemán; y de Marín (2011) y Fábregas y Marín (2015), para el caso de los VPEO en español, que las lecturas distinguidas en 3.1 corresponden a estados (es decir, predicados atélicos no dinámicos).

La pregunta, ahora, es a qué categoría específica de estatividad corresponden los estados causativos y si, en términos empíricos, es suficiente, al menos para el español, la distinción entre predicados IL y SL.

\subsection{Estados IL, SL y EVENTOS NO DINÁMicos}

Como mencionamos en 1.1 y 1.2, al menos desde el trabajo de Pylkkänen (2000) ha pasado a ser común el reconocimiento de los estados causativos, desarticulando, así, el vínculo tradicional entre causatividad y dinamicidad. Sin embargo, mencionábamos allí que, si un estado expresaba una eventualidad de causación, este debía ser de nivel de estadio (SL) y no de individuo (IL), conclusión asumida igualmente por Fábregas y Marín (2015). 
Ampliaremos aquí esta discusión, aduciendo contextos adicionales que mostrarán que la clasificación de los estados causativos como estados SL deriva de una caracterización empírica solo parcialmente adecuada. Para ello, comentaremos dos fenómenos semánticos con claras repercusiones gramaticales: la admisibilidad de lecturas epistémicas orientadas al presente (Soto 2008, Hallman 2009, Jaque 2014, 2016) y la relación de inclusión entre el tiempo de la eventualidad (E) y el tiempo de referencia (R) (Portner 2005, Marín y McNally 2011, Jaque 2014). Ambos contextos servirán para sostener una subclasificación adicional de los estados, entre aquellos que carecen de una variable eventiva, y que incluye tanto a los estados IL como SL, y aquellos que la poseen (llamados estados davidsonianos en Maienborn 2005 , cf. nota 4, supra) y que, en un continuum de menos a más dinamicidad, se aproximan a los eventos dinámicos.

En primer lugar, reiteremos brevemente uno de los argumentos empíricos que pueden aducirse para distinguir entre estados IL y estados SL. Solo los segundos admiten cuantificación temporal, cuestión en que, como es de esperar, coinciden los estados causativos (22) con los predicados introducidos por la cópula estar $(21 \mathrm{~b})^{14}$, la que, según asumiremos en la presente exposición, selecciona predicados SL (cf. Arche 2006):

(23) a. ??Cada vez que Tomás \{tiene 20 años/sabe inglés/es sueco/mide 2 metros\}, sus amigos lo felicitan.

b. Cada vez que Tomás está \{en su casa/enfermo/con visitas\}, recuerda un trámite urgente.

(24) a. Cada vez que una valla \{bloquea/tapa/obstruye $\}$ la entrada, nos metemos por la ventana.

b. Cada vez que los rumores \{molestan/preocupan/agobian/enfadan\} a Susana, envía una carta al periódico.

Como puede apreciarse, tanto los verbos de alternancia instrumental (24a) como los VPEO (24b) admiten cuantificación temporal, en línea con los

14 La selección de la cópula en español involucra excesivas complejidades como para realizar una caracterización adecuada en este lugar. Como es sabido, existen casos en que la cópula ser puede actuar como introductor de un predicado eventivo y admitir, consecuentemente, contextos como los de (21). Así, podemos decir Cada vez que Juan es generoso con sus amigos / infiel a su pareja / simpático con su vecino.... De acuerdo con Arche (2006), en este cambio aspectual influye la configuración sintáctica del SP que el adjetivo toma como complemento, y que permite crear composicionalmente un evento dinámico (una actividad). A efectos de la exposición siguiente, procuraremos emplear los casos prototípicos más claros. 
estados SL (23b) y en contraste con los estados IL (23a). Veamos ahora dos contextos que, al contrario, agrupan a los verbos de alternancia instrumental y a los VPEO con los predicados eventivos, con exclusión de los estados IL y SL.

\subsubsection{Lectura modal epistémica orientada al presente}

Como se ha discutido ampliamente en la bibliografía, los contextos que ofrecen una lectura modal epistémica orientada al presente seleccionan estados (puros) y rechazan cualquier clase de predicado eventivo (Gennari 2002, Soto 2008, Hallman 2009, Lundquist 2012; Jaque 2014, 2016). Según comentaremos más abajo, este contexto sirve para detectar la extensión temporal inherente que puede tener un predicado, frente a la evaluabilidad en instantes que manifiestan los estados. En español, existe una serie de contextos de esta clase, entre los cuales podemos mencionar el futuro sintético en -ré (Gennari 2002, Soto 2008) y la perífrasis deber + inf (Jaque 2014, 2016; cf. Hallman 2009 para el inglés, Lundquist 2012 para el sueco). Consideremos los siguientes ejemplos:

(25) a. Tomás \{tendrá 20 años / sabrá inglés/ será sueco/ pesará mucho\}.

b. Tomás estará \{en su casa / enfermo / con visitas\}.

(26) a. Tomás debe \{tener 20 años / saber inglés / ser sueco / pesar mucho\}.

b. Tomás debe estar \{en su casa / enfermo / con visitas\}.

Los ejemplos de (25) presentan la conducta que, respecto del futuro sintético, manifiestan los estados IL y SL. En ambos casos, la oraciones son ambiguas entre una lectura temporal -el estado de cosas se sitúa con posterioridad al momento de habla- y una lectura epistémica de presente -el hablante manifiesta un bajo compromiso con la verdad de las proposiciones respectivas (v.g. 'Es probable que Tomás sepa inglés'). Un resultado similar se obtiene al emplear la perífrasis deber + inf: las oraciones expresan preferentemente proposiciones modalizadas sobre un estado de cosas presente, y son ambiguas allí donde es pragmáticamente viable obtener una lectura deóntica (v.g. 'Tomás tiene el deber de saber inglés'), que, análogamente a la lectura temporal del futuro sintético, desplaza el eventual cumplimiento del estado de cosas a un intervalo posterior al momento de habla.

Contrástese dicho resultado con lo que se obtiene al emplear predicados eventivos: 
(27) a. Tomás \{aprenderá inglés / se volverá sueco / ganará peso\}.

b. Tomás \{correrá por el parque / cantará / bailará / nadará\}.

En (27a), vemos que los eventos dinámicos télicos rechazan la lectura epistémica y exhiben, en cambio, una lectura temporal excluyente. Una conducta análoga presentan los eventos dinámicos atélicos, como se observa en (27b), que, al menos en lectura no habitual, exigen una interpretación temporal y no modal. El mismo patrón se obtiene al emplear la perífrasis deber + inf, que desencadena una lectura deóntica prospectiva excluyente:

(28) a. Tomás debe \{aprender inglés / volverse sueco / ganar peso\}. b. Tomás debe \{correr por el parque / cantar / bailar / nadar ${ }^{15}$.

Vemos, pues, que la disponibilidad de una lectura epistémica se correlaciona con el aspecto léxico estativo del predicado de base. A efectos, pues, de estos contextos, tanto los predicados IL como SL son igualmente estativos.

El patrón, sin embargo, se rompe al incluir en esta consideración los estados causativos:

(29) a. La valla \{bloqueará / tapará / obstruirá\} la entrada.

b. "La carga tampoco debe obstruir el campo de visión del conductor, ni ocultar luces, catadióptricos ni placas" (google. com [recuperado: 05.01.17]).

(30) a. Los rumores \{molestarán /preocuparán / agobiarán / enfadarán\} a Susana ${ }^{16}$.

b. “Lo debe preocupar la condena a Google por espionaje?” (google.com [recuperado: 06.01.17]).

c. "La costumbre de la masturbación no lo debe preocupar, es algo completamente normal y frecuente en los hombres, fisiológico" (google.com [recuperado: 16.12.16]).

15 Descártese una lectura habitual/genérica (es decir, estativa) del predicado.

16 Para el establecimiento de los juicios de gramaticalidad aquí expuestos, nos restringimos a una estructura oracional en la que el sujeto corresponde a un SD léxico explícito, seguido del verbo y su objeto (el experimentante), construcción en la que, estimamos, el patrón interpretativo indicado se presenta de modo más claro. No obstante, algunos contextos parecen favorecer el acceso a la lectura epistémica con verbos de objeto experimentante. Por ejemplo, si se sustituye el SD explícito por un pronombre indefinido del tipo algo, la lectura epistémica parece aflorar sin problemas: 
Como puede apreciarse en los ejemplos de (29), la lectura temporal o deóntica se impone frente a la lectura epistémica, en claro contraste con lo que exhiben los estados IL y SL, en los que la lectura modal es fácilmente accesible. En este contexto, por lo tanto, los estados causativos difieren de un estado SL prototípico y se aproximan en cambio a los eventos dinámicos, aun cuando, como hemos comprobado en la sección anterior, no lo sean.

Un conjunto de datos que refuerza esta conclusión es el que se obtiene al emplear un verbo causativo no dinámico bajo un contexto gramatical estativizador, como la forma progresiva estar + gerundio $^{17}$. En dicho contexto, la lectura epistémica se vuelve inmediatamente admisible, como se observa en los ejemplos de (31), en los que la lectura deóntica desaparece (el lector puede comprobar que un resultado análogo se obtiene al otorgar al predicado de base una lectura habitual/genérica):

(31) a. "Tienes que abrir la tapa de atrás de la Notebook y limpiar la pelusa que debe estar obstruyendo la salida de aire" (google.com).

b. "A poco de cumplir 21 años, Justin Bieber se desayunó este viernes con una noticia que lo debe estar preocupando" (google. com).

Naturalmente, este patrón vuelve a distanciar a los estados causativos de los estados puros SL o IL, que no requieren (y que, de hecho, tampoco admiten) su inserción bajo la forma progresiva para volver admisible la

i. Algo \{preocupará/debe preocupar\} a Marcelo.

Nótese, sin embargo, que la lectura epistémica va aquí acompañada de una entonación marcada, con énfasis en algo, indicio de que puede tratarse de una focalización. No entraremos en el análisis detallado de este tipo de estructuras. Por otra parte, como comentaremos con mayores detalles en 3.4, la codificación del experimentante en dativo favorece también el acceso a la lectura epistémica (v.g. A Susana le deben molestar los rumores), cuestión que, de hecho, se aplica igualmente al ejemplo de (i), que, con lectura epistémica, se percibe como más natural si el verbo va a acompañado de un clítico de dativo (v.g. Algo le debe preocupar a Marcelo). En este caso, sin embargo, pensamos que se trata de una estructura genuinamente diferente, análoga a la de un verbo de dativo léxico como gustar, verbos que, según propondremos en 3.4 , se comportan como estados puros y no como eventos no dinámicos causativos.

17 El carácter estativo de la forma progresiva ha sido ampliamente discutido en la bibliografía. Sobre este punto, pueden consultarse Vlach (1981), Mittwoch (1988), Parsons (1989), Arche (2006), Hallman (2009). Para el español, Arche (2006) observa la fácil admisión de la paráfrasis estativo/locativa: Estoy escribiendo un artículo $>$ Estoy en ello, cuestión que se condice con el origen léxico estativo, en español y otras lenguas, de las perífrasis de aspecto progresivo (cf. Bybee et al. 1994). 
lectura epistémica (v.gr. *Juan debe estar sabiendo mapudungún, *Andrés debe estar estando en su casa).

Finalmente, la conducta de los estados causativos se asemeja, a este respecto, a la que manifiesta la clase de los llamados estados davidsonianos, es decir, eventualidades no dinámicas a las que se atribuye un argumento eventivo en su representación semántica (Maienborn 2005; Rothmayr 2009, Fábregas y Marín 2013, 2015; Jaque 2014, 2016). Algunos predicados correspondientes a esta categoría incluyen verbos de emisión como brillar o chirriar(32) y verbos inergativos como dormir (33), que denotan la permanencia de un estado en el tiempo. Con estos verbos, obtenemos resultados similares a los que se presentan con estados causativos al aplicar contextos de modalidad epistémica (cf. Jaque 2014, 2016). Así, tanto en (32) como en (33), la lectura epistémica se ve bloqueada, frente a una interpretación temporal prospectiva, sea temporal (a) o deóntica (b):
(32) a. La lámpara brillará.
b. La lámpara debe brillar.
(33) a. Tomás dormirá.
b. Tomás debe dormir.

Con respecto a las razones semántico-estructurales que subyacen al rechazo de las lecturas epistémicas en predicados eventivos, se ha argumentado (Hallman 2009, Lundquist 2012, Jaque 2014, 2016) que esta es sensible a la posibilidad de evaluar un predicado en un punto de tiempo, frente a aquellos que requieren para ello un intervalo (cf. Dowty 1979). Así, los eventos, sean dinámicos o no, necesitan cotejarse con al menos dos instantes sucesivos para decidir si son verdaderos, mientras que, en el caso de los estados, basta para ello con un único instante de inspección en la realidad ${ }^{18}$. Esta diferencia en las condiciones temporales de evaluación se sigue del carácter estrictamente homogéneo de un estado, o lo que es lo mismo, del hecho de que un punto cualquiera del intervalo en el que un estado es válido corresponde a una

18 El experimento mental proporcionado por Dowty (1979: 176-177) para ilustrar esto consiste en imaginar una pelota cayendo por una pendiente: un único instante (captado por una fotografía, por ejemplo) no nos permite decidir si la pelota cae, sube o se mantiene estática, pero una sucesión de dos instantes permite dilucidar el asunto. Asimismo, los estados que él llama de intervalo demandan, igualmente, más de un instante para ser verdaderos o falsos. Siguiendo el mismo ejemplo, para saber si la pelota yace sobre la pendiente, necesitamos saber si se mantiene en el mismo punto del espacio por al menos dos instantes sucesivos. De lo contrario, solo podemos decir que está allí por el instante que le permite, idealmente, el movimiento continuo descendente o ascendente. 
muestra suficiente del predicado en su conjunto, propiedad conocida como subintervalo estricto (Benett y Partee 1978, Taylor 1977, Rothmayr 2009, Marín 2013).

Dado que la lectura modal se orienta, en los contextos ofrecidos, hacia el momento de habla, y asumiendo que este es puntual, solo admitirán una lectura epistémica aquellos predicados que puedan ser evaluados en instantes y no los que solo pueden serlo en intervalos. Una consecuencia de ello es que los estados SL, que, como hemos visto, admiten sin problemas la lectura epistémica, la pierden en cuanto se introduce una modificación adverbial que denote un intervalo:

(34) Tomás estará con visitas \{de dos a tres/un rato\}.

De este modo, los estados SL, según se ejemplifica en (34), se comportan contextualmente como predicados de evento si se añade explícitamente la mención de un intervalo. En otras palabras, estar enfermo puede ser evaluado en instantes, pero estar enfermo de dos a tres solo puede ser cotejado con un periodo extenso de tiempo. De este modo, dado que la lectura modal exige una orientación deíctica al momento de habla, conservamos únicamente la interpretación de futuro. Los predicados de intervalo, sean eventos dinámicos, estados davidsonianos o, como venimos comprobando, estados causativos, no requieren de esta modificación adverbial para bloquear la lectura epistémica, toda vez que parecen ser intrínsecamente extensos ${ }^{19}$. Los contextos de modalidad epistémica muestran, así, una diferencia empírica clara entre estados SL y estados causativos.

\subsubsection{Relación de inclusión entre $E$ y $R$}

Veamos ahora algunas diferencias que se obtienen entre estados SL y estados causativos al considerar las relaciones de inclusión entre el tiempo de la eventualidad (E) y el tiempo de referencia $(R)^{20}$, y que aproximan, nuevamente, los estados causativos a los eventos (incluyendo estados

19 Una consecuencia adicional del carácter intrínsecamente extenso de los estados causativos (entendidos como eventos no dinámicos) es la admisibilidad de la forma progresiva (cf. (31)). Si se entiende que la forma progresiva selecciona un subintervalo puntual del intervalo en el que un evento es válido, es natural que los estados puros la rechacen y los eventos la admitan, incluidos los estados causativos aquí revisados. Para más discusión, véase Hallman (2009), Jaque (2016).

20 Tomamos estos términos del análisis temporal iniciado por Reichenbach (1947) (cf. Klein 1994, Portner 2005). 
davidsonianos y eventos dinámicos). Veremos que, en el caso de los estados

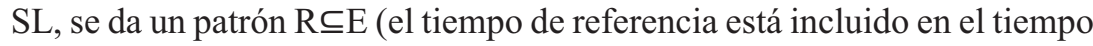
de la eventualidad), mientras que los eventos presentan un patrón $\mathrm{E} \subseteq \mathrm{R}$ (el tiempo de la eventualidad está incluido en el tiempo de referencia). Comentaremos, asimismo, cuál es el correlato semántico al que obedece esta distribución de lecturas.

El primer contexto que exhibe esta distribución de lecturas corresponde a los adverbios de referencia temporal, contexto que adoptamos, con modificaciones, de Marín y McNally (2011) (más detalles en Marín 2013: $85)^{21}$. Los contextos adverbiales que ejercen una cuantificación temporal tienen un efecto de interpretación distinto tratándose de eventos o de estados. Consideremos los ejemplos siguientes ${ }^{22}$ :

(35) a. Siempre que entro en la habitación, Juan está en la cama. b. Siempre que lo visito, Tomás está con su madre.

(36) a. Siempre que entro en la habitación, la cortina \{bloquea/tapa/ obstruye \} la ventana.

b. Siempre que la visito, el problema \{preocupa/agobia/molesta $\}$ a Susana.

(37) a. Siempre que entro en la habitación, la luz brilla.

b. Siempre que entro en la cocina, la ampolleta explota.

c. Siempre que vamos a la plaza, Tomás corre.

En (35), donde encontramos estados SL, vemos que el tiempo de referencia marcado por la subordinada puede estar incluido en el tiempo de la eventualidad (correspondiente al predicado principal): así, Juan puede haber estado en la cama con anterioridad a que entrásemos a la habitación. La opción contraria está, ciertamente, disponible, pero no es la más sobresaliente. Esta relación temporal se invierte en los ejemplos de (36), donde encontramos estados causativos (de alternancia instrumental en (36a), VPEO en (36b)). Existe aquí una fuerte preferencia por una lectura $\mathrm{E} \subseteq \mathrm{R}$, es decir, en que el bloqueo de la cortina está contenido en el intervalo marcado por mi presencia en la habitación, del mismo modo que el estado

21 La prueba, originalmente, es aplicada por los autores a los verbos psicológicos reflexivos (preocuparse, molestarse), para mostrar que se trata de predicados incoativos, si bien estativos.

22 Adviértase que esta prueba no es aplicable con estados puros IL, puesto que implica restringir temporalmente una eventualidad, cuestión que los estados IL rechazan de modo independiente. Así, *Siempre que lo visito, Juan es inteligente/alto/español es semánticamente anómala. 
de preocupación de Susana se inicia durante mi visita y no parece tener vigencia con anterioridad a ella ${ }^{23}$. Esta conducta sigue el patrón de los predicados eventivos ya comentados anteriormente: estados davidsonianos (37a), eventos dinámicos télicos (37b) y eventos dinámicos atélicos (37c). En todos estos casos, el tiempo de la subordinada (R) enmarca al tiempo de la eventualidad (E) del predicado principal.

El segundo contexto que revisaremos aquí corresponde a la interpretación que muestran algunos tiempos gramaticales (Jaque 2014, 2016). Como observa Portner (2005: 144), el pasado inglés ofrece dos lecturas: $E \subseteq R$ y $\mathrm{R} \subseteq \mathrm{E}$, que se correlacionan con la distinción entre evento y estado, respectivamente (cf. Klein 1994, Katz 2000, entre otros). Así, encontramos contrastes como el siguiente, tomado de Katz (2000: (52)-(54)):

(38) a. Peter drank a beer (??and he is still drinking one).

b. Peter walked around aimlessly (??and he still is).

c. Peter was sick (and he still is).

Mientras que, en (38a-b), los eventos de beber una cerveza o de pasear quedan incluidos en el tiempo de referencia (que, en este caso, es anterior al presente), el ejemplo de (38c), en que empleamos un estado, manifiesta una relación inversa. Así, no es el caso que Peter pueda seguir bebiendo una cerveza ni que siga paseando, mientras que sí es perfectamente posible que siga estando enfermo. Nótese que, tal como sucede con la prueba anterior, este contraste depende de la oposición estado/evento y no de la distinción télico/atélico. Así, (38b), que es un predicado atélico, pero eventivo, bloquea igualmente la lectura $\mathrm{R} \subseteq \mathrm{E}$.

En español, sin embargo, la correlación entre lectura $E \subseteq R$ y eventos, por una parte, y lectura $\mathrm{R} \subseteq \mathrm{E}$ y estados, por otra, no se cumple de modo generalizado, puesto que el sistema verbal español cuenta con mecanismos aspectuales independientes para fijar una $u$ otra lectura, sin hacerla descansar, por lo tanto, en la naturaleza aspectual del predicado léxico:

23 En 3.4 volveremos sobre la disponibilidad de la relación temporal inversa, al considerar los VPEO de dativo, que se comportan como estados puros SL ante esta prueba. Considérese que los VPEO alternan productivamente entre el acusativo y el dativo (v.g. preocuparle/lo), cambio que se correlaciona con una diferencia aspectual, como ha sido tradicionalmente observado (cf. Strozer 1976, Arad 1999, Marín 2015, entre otros). 


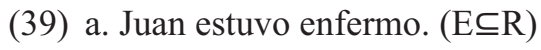

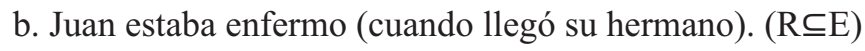

Como podemos observar en (39), las formas de pasado del español nos "obligan" a adoptar una lectura $\mathrm{C} \subseteq \mathrm{R}$, si elegimos el indefinido (39a); o una lectura $\mathrm{R} \subseteq \mathrm{E}$, si elegimos el imperfecto (39b). No obstante, el futuro simple sí permite reproducir el fenómeno que, para el pasado, se aprecia en los ejemplos ingleses de (38), una vez que nos restringimos a la lectura temporal y desechamos la lectura modal epistémica que,como hemos comentado más arriba, se encuentra disponible en predicados estativos. Así, consideremos los ejemplos de (40) bajo una lectura estrictamente temporal, es decir, donde $\mathrm{R}$ es posterior al presente de habla:

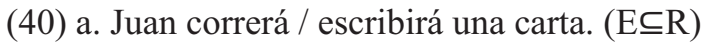

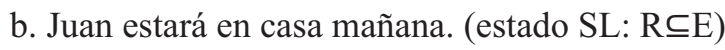

En estos casos, vemos que la distribución de las lecturas $E \subseteq R$ y $R \subseteq E$ vuelve a correlacionarse con la distinción estado/evento. De este modo, en (40a) tanto el evento de correr como el de escribir una carta quedan contenidos en el tiempo de referencia: no puede ser el caso que Juan haya comenzado ya a correr o a escribir una carta. En cambio, en (40b), donde encontramos un estado puro de tipo SL, el tiempo dereferencia está incluido en el tiempo de la eventualidad, de forma tal que la oración puede ser cierta en un contexto donde Juan se encuentra ya en casa en la actualidad (y seguirá allí hasta mañana). De modo interesante, los estados causativos (41) vuelven a replicar el patrón de los estados davidsonianos (42), que sigue a su vez el de los eventos dinámicos $(40 \mathrm{a})$ :

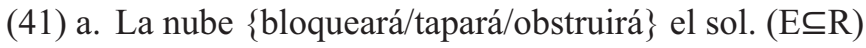

b. El indicador de popularidad \{preocupará/molestará/agobiará\} a

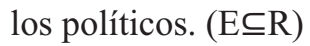

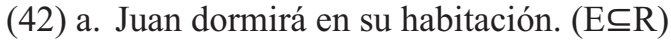

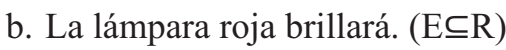

De este modo, tanto los verbos de alternancia instrumental (41a) como los

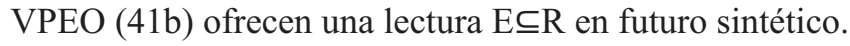

Los datos precedentes pueden derivarse de la presencia o ausencia de una variable eventiva apelando al denominado requisito de maximalidad (maximality requierement, Katz 2000: 411). De acuerdo con Katz, los eventos, a diferencia de los estados, deben interpretarse empleándose de 
forma máxima, es decir,si localizamos un evento respecto de un marco de referencia temporal, debemos localizar el evento en su totalidad. Ya Herweg (1991, apud Katz 2000: 410) había observado que si los estados contuviesen un argumento subyacente análogo al que se postula para representar un evento, el contraste que ofrece el pasado inglés (y el futuro simple en español) se tornaría gratuito. En ambos casos, diríamos que el evento $e$ o el estado $s$ se localizan en el pasado, pero no se apreciaría una razón clara para obtener

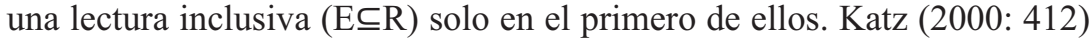
formula el requisito de maximalidad como parte del esquema interpretativo de una predicación de evento:

(43) $\exists e ~[P(e)]=\{<m, t>\mid$ hay un evento máximo del tipo $P$ en el mundo $\mathrm{m}$ en el tiempo $\mathrm{t}\}$

De este modo, si existe un evento de escribir una carta, correr, brillar o bloquear, que se localiza en un mundo y en un tiempo determinados, el evento en su totalidad debe situarse en las coordenadas expresadas en dicho índice. Tratándose de predicados que no incluyen en su representación una variable eventiva, el requisito de (43) no encuentra aplicación. En palabras de Katz (2000: 413):

Since in the logical form of state sentences there is no reference to underlying states, there is no maximality requirement on "states", but since in the logical form of event sentences there is existencial reference to an underlying event, there is a maximality requirement on the event thus referred to.

Por lo tanto, podemos sostener que la conducta de los estados causativos frente a los adverbios de referencia temporal y las lecturas del futuro simple en su lectura no epistémica avalan la idea de que esta clase de verbos pertenece al grupo de los eventos (si bien no dinámicos), es decir, aquellos que poseen una variable eventiva en su representación semántica. Adoptando la categoría introducida en Maienborn (2005), proponemos que se trata, pues, de estados davidsonianos.

Diremos que aquellos predicados que admiten lecturas epistémicas orientadas al presente, así como lecturas temporales no inclusivas $\mathrm{R} \subseteq \mathrm{E}$, corresponden a estados puros (Jaque 2014, 2016), clase que, según hemos venido motivando empíricamente, incluye tanto a los estados IL como a los estados SL (cualquiera sea la diferencia semántico-sintáctica ulterior que autorice esta subclasificación). Por otra parte, la categoría de los eventos poseerá, como características generales, las propiedades opuestas: rechazo de lecturas epistémicas y lecturas temporales inclusivas. Dicha categoría 
englobará los estados causativos, perteneciente a la clase de los estados davidsonianos, junto a los eventos dinámicos (télicos y atélicos). Vemos, así, que aunque existan propiedades comunes a estados causativos y estados SL, se trata de un solapamiento parcial y no total. Una síntesis de los resultados alcanzados en 3.3.1 y 3.3.2, y que amplían los que se describen en 3.2, se ofrece en la siguiente tabla ${ }^{24}$ :

\begin{tabular}{|c|c|c|c|c|c|c|c|}
\hline \multirow{3}{*}{$\begin{array}{l}\text { Propiedad } \\
\text { semántica }\end{array}$} & \multirow{3}{*}{$\begin{array}{l}\text { Contexto } \\
\text { gramatical }\end{array}$} & \multicolumn{2}{|c|}{ Estados puros } & \multicolumn{4}{|c|}{ Eventos } \\
\hline & & \multirow{2}{*}{\begin{tabular}{|c|}
$\mathrm{IL}$ \\
$($ ser \\
sueco $)$ \\
\end{tabular}} & \multirow{2}{*}{$\begin{array}{c}\text { SL } \\
\text { (estar } \\
\text { enfermo) }\end{array}$} & \multicolumn{2}{|c|}{$\begin{array}{c}\text { Estados } \\
\text { davidsonianos }\end{array}$} & \multicolumn{2}{|c|}{ Dinámicos } \\
\hline & & & & $\begin{array}{l}\text { Causativos } \\
\text { (bloquear, } \\
\text { preocupar) }\end{array}$ & $\begin{array}{c}\text { No } \\
\text { causativos } \\
\text { (brillar) }\end{array}$ & $\begin{array}{l}\text { Atélicos } \\
\text { (correr) }\end{array}$ & $\begin{array}{l}\text { Télicos } \\
\text { (hundir) }\end{array}$ \\
\hline \multirow[b]{2}{*}{ Telicidad } & en $x$ tiempo & $(-)$ & $(-)$ & - & - & - & + \\
\hline & $\begin{array}{l}\text { Una vez + } \\
\text { participio }\end{array}$ & $(-)$ & $(-)$ & - & - & - & + \\
\hline \multirow{2}{*}{ Dinamicidad } & Lentamente & $(-)$ & $(-)$ & - & - & + & + \\
\hline & Parar de + inf & $(-)$ & $(-)$ & - & - & + & + \\
\hline $\begin{array}{l}\text { Lectura } \\
\text { episódica }\end{array}$ & $\begin{array}{l}\text { Cuantificación } \\
\text { temporal }\end{array}$ & - & + & + & + & $(+)$ & $(+)$ \\
\hline $\begin{array}{l}\text { Extensión } \\
\text { temporal } \\
\text { inherente }\end{array}$ & $\begin{array}{l}\text { Lectura modal } \\
\text { epistémica }\end{array}$ & + & + & - & - & - & - \\
\hline $\begin{array}{l}\text { Variable } \\
<\mathrm{e}>\end{array}$ & $\begin{array}{l}\text { Lectura } \\
\text { temporal } \mathrm{E} \subseteq \mathrm{R}\end{array}$ & - & - & + & + & + & + \\
\hline
\end{tabular}

Tabla 2. Caracterización gramatical de estados puros, estados davidsonianos y eventos dinámicos

\subsection{Algunas diferencias entre VPEO de Dativo y acusativo}

En los apartados anteriores, al tratar los VPEO, nos hemos restringido exclusivamente a los que seleccionan acusativo, puesto que asumimos que son estos predicados los que expresan realmente un significado causativo

${ }^{24}$ Los valores entre paréntesis (-/+) indican resultados no discutidos en el presente trabajo, pero que podemos asumir a efectos de la presentación. 
(cf. Pesetsky 1995, Arad 1999, Landau 2009, Marín 2015). En esta sección, quisiéramos contrastar brevemente la conducta que hemos aislado en estos predicados respecto de lo que Belletti y Rizzi (1988) llaman clase III, es decir, VPEO que codifican el experimentante en dativo. La asunción tradicional consiste en correlacionar caso dativo con aspecto estativo y caso acusativo con aspecto dinámico. $\mathrm{Si}$, como vemos, también los VPEO de acusativo son "estativos", ¿lleva asociada, con todo, una diferencia aspectual/argumental la alternancia de caso en que se expresa el experimentante?

Existe un restringido grupo de verbos que solo toman dativo (gustar, disgustar, doler, apetecer, importar), frente a un extenso grupo que alterna productivamente entre dativo y acusativo (preocupar, molestar, agobiar, asustar, apenar, asombrar, cabrear) (cf. Marín 2015), entre los cuales se cuentan los que hemos usado, considerando su manifestación con acusativo, para los ejemplos analizados en los apartados precedentes. Existen diversas diferencias entre las construcciones en que el experimentante se codifica en dativo (esté determinado léxicamente a ello o no) y aquellas en que lo hace en acusativo. Considérense los ejemplos siguientes:

(44) a. A Juan le gustan los rumores.

b. Los rumores preocupan a Juan / lo preocupan.

Respecto de (44a), no tiene mucho sentido formular los rumores como "causa" del gusto sentido por Juan; se trata, más bien, de un Tema. Consideramos la eventualidad como una disposición de Juan hacia un cierto objeto, antes que como el ejercicio de una influencia desde los rumores hacia Juan. En cambio, en (44b) no es Juan el que siente una cierta inclinación hacia los rumores, sino ellos los que generan en él un cierto estado mental, vigente solo mientras estos conserven una cierta disposición episódica (cf. Arad 1999).

Resulta de interés destacar, pues, que las configuraciones del tipo de (44a) se comportan como estados puros y no como eventos no dinámicos, al menos respecto de los criterios empleados más arriba. Así sucede tanto con los VPEO alternantes cuando toman dativo como con los verbos de dativo léxico: 
(45) a. A Diego debe \{gustarle/importarle\} su familia.

b. Siempre que lo visito, a Diego le gusta un autor diferente.

(46) a. A Susana debe \{preocuparle/molestarle/agustiarle\} su situación laboral.

b. Siempre que la visito, a Susana \{le preocupa/molesta/angustia\} su situación laboral.

Vemos, así, que la disponibilidad de lecturas epistémicas orientadas al presente se encuentra disponible tanto para verbos de experimentante dativo léxico (45a) y alternante (46a) (v.g. 'Es probable que a Diego le guste su familia', 'Es probable que a Susana le moleste su situación laboral', cf. (30b) “'Lo debe preocupar la condena a Google por espionaje?”, con lectura deóntica/prospectiva excluyente). Por otra parte, al insertar un contexto adverbial de referencia temporal, en ambos casos (ejemplos (b)) obtenemos una relación de inclusión temporal $\mathrm{R} \subseteq \mathrm{E}$ (así, el inicio de la preocupación de Susana por su situación laboral puede ser perfectamente previo a mi visita).

Es importante destacar el patrón anterior, en el marco de la presente propuesta, al menos por dos razones. La primera es que, en muchos casos, la interpretación de los VPEO de acusativo puede ser inestable, o menos clara que la que se obtiene al emplear verbos de alternancia instrumental del tipo bloquear. Al dilucidar los juicios de gramaticalidad, conviene tener presente, pues, que los verbos del tipo preocupar alternan entre una configuración causativa y una configuración estativa pura análoga a la de gustar, cuestión que se correlaciona con el caso asumido por el experimentante ${ }^{25}$. En segundo

25 Esta correlación entre aspecto y caso resulta más clara en zonas dialectales no "laístas", es decir, donde una secuencia como La dijo que... se juzga como agramatical. Si la correlación entre, por un lado, CAUSA y evento no dinámico y, por otro, TEMA y estado puro es correcta, los hablantes que atribuyan fácilmente una lectura epistémica a secuencias como $\boldsymbol{L} \boldsymbol{o}$ debe preocupar la economía (o con la misma facilidad con que la atribuirían a un ejemplo como $A$ Juan le debe preocupar la economía) seguramente posean una distribución de caso diferente. En ejemplos de este tipo,esperaríamos una conceptualización del SD la economía más cercana a un tema que a una causa, junto a una preferencia por el orden canónico posverbal que este argumento recibe en los VPEO de dativo (cf. Le gusta el cine, no marcado, frente a El cine le gusta, opción informativamente marcada). No podemos abordar una discusión más detallada de este intrincado aspecto, que involucraría un estudio de variación dialectal más allá de los objetivos de la presente investigación.

Por otra parte, nótese que la intervención aspectual del caso es un fenómeno conocido en otras lenguas y advertido por diversos autores. Así, en alemán, ciertas preposiciones alternan entre caso dativo (Ich bin im ${ }_{\mathrm{DAT}}$ Kino 'estoy en el cine') y acusativo (Ich gehe ins ${ }_{\text {Acus }}$ Kino 'voy al cine') para marcar una distinción aspectual entre estado y evento dinámico, respectivamente. En finés, se ha observado igualmente la distribución de los casos partitivo y acusativo en 
lugar, es interesante observar que el mayor o menor grado de estatividad exhibido por los VPEO está en correlación con el papel temático que se asigna al sujeto. Solo cuando este es CAUSA, es admisible la inclusión de la estructura verbal subyacente en el grupo de los eventos no dinámicos, no así cuando es TEMA. Como quisiéramos avanzar en el apartado siguiente, dicho cambio en la estructura argumental de los predicados correspondería también a una estructura sintáctica distinta.

\section{LA SEMÁNTICA DEL CAMBIO Y LA PERMANENCIA Y SU EXPRESIÓN SINTÁCTICA}

Para finalizar este estudio, quisiéramos presentar cómo encajan los datos presentados en las secciones anteriores con un modelo de representación semántica que puede encontrar un correlato de forma más o menos natural en la sintaxis verbal (cf. Jaque 2014, 2016). En particular, consideraremos la formalización de las nociones de cambio y causación que realiza Moreno Cabrera (2003) y que, como veremos, tiene igualmente su expresión en distintas propuestas recientes sobre el análisis de los subcomponentes de la frase verbal (cf. Mateu 2002, Ramchand 2008), que permiten computar sus posibilidades aspectuales y argumentales. Por supuesto, esta sección no pretende ofrecer una solución definitiva ni exhaustiva a los problemas que plantea la expresión sintáctica de la estructura argumental y la estructura eventiva, sino solo proporcionar un mecanismo explícito (si bien tentativo) a través del cual dar cuenta de los patrones gramaticales examinados en los apartados anteriores. Al término de la exposición, se ponderan algunas consecuencias del análisis no contempladas hasta ahora, que permiten otorgarle valor predictivo.

De acuerdo con Moreno Cabrera (2003), los tipos de suceso se articulan sobre la noción de estado como bloque elemental. Un estado se define como una relación $(E)$ entre una entidad $(x)$ y una propiedad $(F)$ (adaptamos las letras iniciales por claridad de la exposición):

correlación con un valor atélico y télico, respectivamente (cf. Borer 2005). De este modo, no es extraño que el caso que codifica el experimentante en español marque una cierta distinción entre grados de dinamicidad análoga a la que (si bien situando el límite en puntos diferentes de la escala) observamos en otras lenguas. 


\section{(47) $\mathbf{E}(x, F)$}

La relación entre dos o más estados produce lo que Moreno Cabrera denomina Proceso, y que funcionará, igualmente, como un predicado específico $(P)$ cuyos argumentos corresponden al tipo de suceso básico, es decir, el estado. Si existe una relación de oposición entre los estados vinculados por $\mathrm{P}$, hablaremos de mutaciones, en las que el paso de un estado a otro coincide con la adquisición (o pérdida) de una propiedad por parte de una entidad; si, en cambio, $P$ relaciona dos o más estados idénticos, hablaremos de mantenimientos, en los que una entidad sigue relacionada con una cierta propiedad. Estas posibilidades se ejemplifican a continuación:

$$
\begin{array}{rll}
\text { (48) a. } \mathbf{P}[\neg \mathbf{E}(\mathrm{x}, \mathrm{F}),(\mathbf{E}(\mathrm{x}, \mathrm{F})] & \text { MUTACión } \\
\text { b. } \mathbf{P}[\mathbf{E}(\mathrm{x}, \mathrm{F}),(\mathbf{E}(\mathrm{x}, \mathrm{F})] & \text { MANTENimiento }
\end{array}
$$

Finalmente, cada una de las estructuras de (43) puede funcionar como segundo argumento de un último predicado, Acción $(A)$, que relaciona entidades $(y)$ con Procesos, dando lugar a estructuras agentivas. Para la relación entre una entidad y un proceso de mutación, el autor reserva el término modificación, aunque no se encuentra un término análogo para el caso donde el Proceso adoptado por A como segundo argumento corresponda a un mantenimiento. Las estructuras respectivas serían las siguientes:

$$
\begin{aligned}
& \text { (49) a. } \mathbf{A}(\mathrm{y}, \mathbf{P}[\neg \mathbf{E}(\mathrm{x}, \mathrm{F}),(\mathbf{E}(\mathrm{x}, \mathrm{F})]) \\
& \text { b. } \mathbf{A}(\mathrm{y}, \mathbf{P}[\mathbf{E}(\mathrm{x}, \mathrm{F}),(\mathbf{E}(\mathrm{x}, \mathrm{F})])
\end{aligned}
$$

MODIFICACIÓN

MANTENIMIENTO (CAUSADO)

Para ejemplificar la correspondencia de estas estructuras con predicados reales del español, considérense las oraciones siguientes y sus correspondientes estructuras semánticas, en las que las variables de las fórmulas anteriores se sustituyen por expresiones concretas ${ }^{26}$ :

26 Por supuesto, las unidades léxicas que se emplean para sustituir variables por elementos concretos no deben asimilarse literalmente al repertorio de categorías léxicas de una lengua en particular. Así, podemos escribir abierta o apertura, sin que el valor de la fórmula se altere sustancialmente. 
(50) a. La puerta está abierta

E(la puerta, abierta)

b. La puerta se mantiene abierta

$\mathbf{P}[\mathbf{E}$ (la puerta, abierta), $\mathbf{E}$ (la puerta, abierta) $]$

c. Juan mantiene la puerta abierta

$\mathbf{A}($ Juan, $\mathbf{P}[\mathbf{E}$ (la puerta, abierta), $\mathbf{E}($ la puerta, abierta) $])$

Nótese que, de acuerdo con las asunciones del autor, el papel temático de agente posee un valor estrictamente relacional: corresponde al primer argumento de una función que toma como segundo argumento un proceso. No necesitamos concebir al individuo en cuestión como una entidad volitiva o dotada de control mental. De este modo, existe cierto colapso formal entre los papeles temáticos de CAUSA y AGENTE, en la medida en que ambos pueden considerarse como entidades que condicionan la puesta en marcha de un proceso. Dicha solución es similar a la que se adopta en distintos modelos actuales de descomposición de la frase verbal, en los que una proyección funcional (SVoz en Kratzer 1996, Sv en Chomsky 2001, SInicio en Ramchand 2008, o SEvento en Borer 2005) proyecta en su especificador un argumento externo al que se asigna un papel temático genérico: INICIADOR (por ejemplo, en Ramchand 2008) o Factor de Contribución Crucial (CCF, por sus siglas en inglés, en Neeleman y van de Koot 2012), que puede especificarse composicionalmente como agente, instrumento o causa en función de las características globales de la estructura (cf. Cuervo 2015).

Ahora bien, la propuesta de Moreno Cabrera (2003) tiene directo interés para el análisis que hemos realizado en este trabajo. Recapitulando, hemos sostenido que los estados causativos, a pesar de carecer de dinamicidad, no pueden equipararse a estados puros, sean estos IL o SL, sino que deben ser tratados como eventos. Una de las consecuencias de ello es que los estados causativos, según se aprecia en la tendencia a rechazar lecturas epistémicas orientadas al presente, no pueden ser evaluados en puntos de tiempo, sino que abarcan necesariamente un intervalo (a pesar de que el contenido de ese intervalo corresponda a una situación homogénea). Así, está preocupado puede ser válido en cualquier punto de tiempo, mientras que la economía lo preocupa involucra un desarrollo en el tiempo. La propuesta semántica de Moreno Cabrera capta esta diferencia de forma directa, toda vez que, para que exista causación, es decir, para que podamos introducir una entidad que se considere como "agente" (o causante) de un suceso, debemos tener primero una relación entre estados sucesivos, con independencia de que estos 
conformen una mutación o un mantenimiento. No existe, por definición, una relación directa entre agentes y estados (puros) ${ }^{27}$.

De modo crucial, si tenemos sucesión de estados, introducimos necesariamente extensión temporal. Como consecuencia de esta extensión, el predicado puede ser evaluado únicamente en intervalos y no en puntos de tiempo. La similitud de efectos gramaticales que se presentan entre estados davidsonianos no causativos (del tipo brillar) y en estados davidsonianos causativos (del tipo bloquear o preocupar) (cf. 3.3), se sigue de que, en efecto, ambos tipos de predicado compartirían la misma estructura básica. Dicho en otras palabras, un estado davidsoniano (o evento no dinámico) se puede modelar como un Proceso de mantenimiento en la teoría semántica de Moreno Cabrera, en la que P relaciona una serie indefinida de estados idénticos. Un estado davidsoniano, así entendido, corresponderá a una parte integrante de un estado causativo:

(51) a. La lámpara brilla.

$\mathbf{P}[\mathbf{E}$ (lámpara, brillo), ..., E(lámpara, brillo)]

b. La roca bloquea la entrada.

$\mathbf{A}($ la roca, $\underline{\mathbf{P}}[\mathbf{E}$ (la entrada, bloqueo), ... $\underline{\mathbf{E}}$ (la entrada, bloqueo)]

Al observar las estructuras semánticas introducidas en (51), se advierte que, en efecto, una paráfrasis adecuada de las oraciones correspondientes sería, para (51a), la lámpara se mantiene con brillo/brillante y, para (51b), la roca mantiene la entrada bloqueada.

La adopción de este modelo, así, explica de manera adecuada el patrón de interpretación temporal que siguen los estados davidsonianos (incluidos los causativos) ante los contextos de modalidad epistémica. Dado que involucran un intervalo, no pueden orientarse al presente en contextos que

27 Es también importante destacar que el operador A relaciona una entidad, no con un evento causante, sino directamente con el proceso que esta pone en marcha. Esta decisión no es trivial, ya que es comúnmente asumido que, desde un punto de vista cognitivo y conceptual, la causación consiste en una relación entre dos eventos, y no entre un individuo y un evento. Sin embargo, existe también cierto consenso en que la expresión lingüística de la causalidad corresponde a una versión empobrecida de esta relación. De modo crucial, aunque los sujetos de verbos causativos pueden denotar eventos, normalmente denotan individuos, sin que puedan aducirse diagnósticos gramaticales claros para la existencia de un subevento de causación implícito en la representación semántica del predicado (como sí puede hacerse, en contraste, con la existencia de estados resultantes en predicados de cambio). Para más discusión sobre este punto, véase Neeleman y van de Koot(2012), Cuervo (2015). Para un propuesta en la que sí existe, en términos lingüísticos, un evento causante (se proyecte o no un argumento externo), véase Pylkkänen (2002, especialmente, cap. 3). 
los obligan a ello, promoviendo una lectura desplazada hacia el futuro (v.g. La roca bloqueará la entrada, con lectura temporal excluyente). El modelo proporciona, pues, un sistema en el que este patrón temporal se sigue de modo natural a partir de la propia definición de agente, al restringir su combinación a procesos (de mantenimiento o de mutación) y bloquear su combinación directa con estados.

¿Qué hay, pues, del segundo grupo de fenómenos que hemos considerado

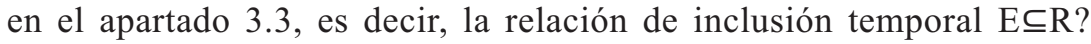
Basándonos en Katz (2000), hemos deducido ese fenómeno de la presencia de un argumento eventivo $<\mathrm{e}>$. Intentaremos ahora conciliar esta propiedad semántica con los aspectos fundamentales de la teoría de Moreno Cabrera (2003) arriba expuesta. Para ello, adoptaremos un modelo de descomposición verbal en el que la sintaxis tiene directa incidencia en las opciones aspectuales y argumentales de los predicados (cf. para una discusión detallada, Jaque 2014, 2016). Es común asumir, en la bibliografía reciente, que la frase verbal comprende, no un único núcleo léxico, sino una secuencia de proyecciones sintácticas ordenadas. Las proyecciones asociadas al significado verbal que computan su tipo de situación y su red argumental marcan una primera fase de derivación sintáctica (Chomsky 2001, Ramchand 2008), en la que se identifican comúnmente tres niveles estructurales:

- Un nivel de predicación básica que relaciona argumentos con propiedades (Sr en Mateu 2002, SResultado en Ramchand 2008, SRaíz en Harley 2009, SRelación en Brucart 2010).

- Un nivel que aporta el significado eventivo básico (SProceso en Ramchand 2008, SAsp en Borer 2005, Sv en Harley 2009).

- Un nivel en el que se introduce un argumento externo, que englobaría los papeles temáticos de CAUSA y AGENTE (SVoz en Kratzer 1996, Sv en Chomsky 2001, SEvento en Borer 2005, SInicio en Ramchand 2008, cf. Cuervo 2015).

Para efectos del presente trabajo, asumiremos, así, una articulación del sintagma verbal en tres niveles, atribuyendo al nivel intermedio, etiquetado aquí como $\mathrm{SV}$, la introducción del argumento eventivo $<\mathrm{e}>$, siguiendo propuestas formuladas en Fábregas y Marín (2013). En tanto, el nivel básico, SR(elacional), podrá adoptar un valor de COINCIDENCIA CENTRAL (CC) o de COINCIDENCIA terminal (CT) (cf. Hale 1984, Mateu 2002, Brucart 2010), que permitirá computar el valor dinámico o estático de la eventualidad. Finalmente, el nivel más externo (Sv) introducirá, siguiendo la asunción tradicional, un argumento externo: 
(52) $\left[_{\mathrm{SV}} \mathrm{SD}_{\text {ARG EXT }} \mathrm{V}^{\mathrm{o}}\left[\mathrm{SV}_{\mathrm{SV}} \mathrm{V}_{<\mathrm{e}}^{\mathrm{o}}\left[\mathrm{SR}_{\mathrm{SR}} \mathrm{SD}_{\text {ARG INT }} \mathrm{R}_{\mathrm{CT} / \mathrm{CC}} \mathrm{SX}\right]\right]\right]$

El sintagma SR tomará como complemento una propiedad y establecerá, de este modo, una relación predicativa con el SD ensamblado en su especificador. Ahora bien, tal como se presenta en Jaque (2014, 2016), asumiremos que el argumento eventivo $<\mathrm{e}>$ introducido en $\mathrm{V}$ posee extensión temporal inherente. De este modo, los eventos se conciben como "trozos de tiempo" en los que puede o no tener lugar un cambio de estado, contenido que vendrá especificado por la proyección predicativa básica. Puesto en términos algo más formales, diremos que, según se expresa en el postulado de (53), para todo evento, la huella temporal de dicho evento corresponde a un intervalo (por oposición a un punto de tiempo):

(53) $\forall \mathrm{e}(\tau(\mathrm{e})=\mathrm{I})$

Al introducir SR como complemento de V, la predicación básica allí expresada adquiere extensión temporal. En otras palabras, el trozo de tiempo contenido en $<\mathrm{e}>$ cubre o expande la relación predicativa introducida en SR. Dicha estrategia se formaliza en (54) (Jaque 2014, 2016):

$$
\begin{aligned}
& \text { (54) a. }\|\mathrm{SR}\|=\mathrm{R}(\mathrm{j})(\mathrm{i}) \\
& \text { b. } \left.\left\|\mathrm{V}^{\mathrm{o}}\right\|\right\rangle=\lambda \mathrm{p} \lambda \mathrm{e} . \mathrm{P}(\mathrm{e}) \& \forall \mathrm{I}[\operatorname{darse}(\mathrm{e}, \mathrm{I}) \rightarrow \operatorname{darse}(\mathrm{p}, \mathrm{I})] \\
& \text { c. }\|\mathrm{SVV}\|=\lambda \mathrm{e} . \mathrm{P}(\mathrm{e}) \& \forall \mathrm{I}[\operatorname{darse}(\mathrm{e}, \mathrm{I}) \rightarrow \operatorname{darse}(\mathrm{R}(\mathrm{j})(\mathrm{i}), \mathrm{I})]
\end{aligned}
$$

La proposición expresada en SR (relación entre un elemento i y uno j) corresponde al aducto de la función expresada en V. En ella, se define una propiedad de eventos tales que, para un evento del tipo P (donde P se sustituye por la unidad léxica verbal de que se trate), se cumple que, para todo intervalo, si el evento se da en ese intervalo, entonces también se da la proposición denotada por SR. (54c) representa la denotación del sintagma SV (la propiedad de eventos en cuestión) una vez que el núcleo $\mathrm{V}^{\mathrm{o}}$ se ha combinado con SR.

Al especificar el valor de R, podemos obtener dos escenarios. Si R adopta un valor de coincidencia terminal, el evento denotará un cambio de estado, toda vez que la relación entre el argumento en posición de especificador de $\mathrm{R}$ y la propiedad introducida en su complemento conforma una trayectoria abstracta, que se entenderá, puesta en el decurso temporal, como un evento 
de adquisición de propiedad (v.g. romperse) ${ }^{28}$. En cambio, si R adopta un valor de coincidencia central, introducimos una condición de homogeneidad: el argumento y su propiedad no se posicionan de modo externo el uno con el otro, sino que los hacemos coincidir en una relación (abstracta) de continente/ contenido (cf. Marín 2013: 57, Hale y Keyser 2002). Este segundo escenario es el que correspondería a un estado davidsoniano, ejemplificado en (55b):
a. $\left[\mathrm{SV}_{\mathrm{SV}} \mathrm{V}_{<\mathrm{e}}\left[{ }_{\mathrm{SR}}\right.\right.$ el vidrio $\left.\left.\mathrm{R}_{\mathrm{CT}} \mathrm{ROTO}\right]\right]$
el vidrio se rompe
b. $\left[\mathrm{SV}_{\mathrm{SV}} \mathrm{V}_{<\mathrm{SR}}\left[\right.\right.$ la lámpara $\left.\left.\mathrm{R}_{\mathrm{cc}} \mathrm{BRILLO}\right]\right]$
la lámpara brilla

Esta manera de modelar un estado davidsoniano (evento no dinámico) puede entenderse, así, como una traducción sintactista de la formalización semántica de un proceso de mantenimiento llevada a cabo por Moreno Cabrera, en la que, grosso modo, SR corresponde a su operador Estado y V corresponde al operador Proceso ${ }^{29}$ :
(56) a. $\left[\left[_{S V} V_{<e>}\right.\right.$
b. $P$
$\left.\left[\mathrm{SR}, \mathrm{R}_{\mathrm{cc}} \quad\right]\right]$
$[\mathrm{E}(\mathrm{x}, \mathrm{F}), \ldots, \mathrm{E}(\mathrm{x}, \mathrm{F})]$

Ahora bien, existen algunas diferencias importantes entre ambos tipos de estructura. En primer lugar, la extensión temporal inherente del predicado, que en la opción semántica de (b) se modela como una secuencia de estados diversos (si bien cualitativamente idénticos) corresponde a una única relación predicativa en la sintaxis (56a). Dicha extensión temporal se atribuye, ahora, a la variable eventiva introducida por V. Así, en lugar de multiplicar los estados en el tiempo, decimos que un mismo estado adquiere "grosor temporal" al ser considerado como el contenido predicativo de un evento. En segundo lugar, y retomando la pregunta que expresábamos más arriba, la introducción de $<\mathrm{e}>$ permite, asimismo, dar cuenta de la segunda

28 En Brucart (2010) se explora una de las posibilidades teóricas que el sistema deja abierta: una estructura SR de coincidencia terminal (CT) no dominada por V (o una proyección eventiva análoga). Esta estructura correspondería a oraciones copulativas de tipo SL, donde estar valúa el rasgo $[+\mathrm{CT}]$ del núcleo de $\mathrm{SR}$ : [ estar $\left[_{\mathrm{SR}}\right.$ él $\mathrm{R}_{[+\mathrm{CT}]}$ contento]]. En términos conceptuales, los predicados copulativos con estar denotarían, justamente, "trayectorias abstractas". En nuestros términos, dichas trayectorias abstractas adquieren el rango de procesos de adquisición una vez que están dominadas por una variable eventiva.

29 Esta equiparación entre sintaxis y semántica no es, en absoluto, una novedad de este trabajo, y se encuentra ya declarada por el propio autor, quien indica que el modelo desarrollado por Mateu (2002), y en el que se inspira en buena medida el análisis tripartito de la frase verbal aquí adoptado, puede entenderse como una contraparte sintáctica de su modelo semántico (Moreno Cabrera 2003: 180 y ss.). 
propiedad semántica que estados davidsonianos comparten con estados

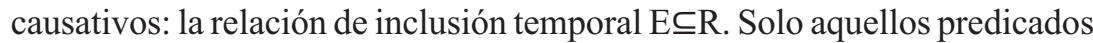
que incluyan un argumento eventivo poseerán obligatoriamente una lectura temporal máxima (Katz 2000). Conservamos, pues, las ventajas empíricas que habíamos reconocido en el modelo semántico de Moreno Cabrera (2003), pero las hemos traducido a un sistema más simplificado que nos permite dar cuenta de una gama más amplia de hechos.

Finalmente, tanto a la estructura de (56a) como a la de (56b) puede añadirse un agente: en la sintaxis (56a), mediante la inserción de Sv; en su correlato semántico (56b), si P [...] es tomado como segundo argumento de un predicado de Acción (A). Respecto de la estructura semántica, decíamos que el carácter eventivo de los estados causativos se seguía de la restricción formal que impedía al operador A relacionar entidades directamente con estados. En términos sintácticos, podemos formular una restricción similar, indicando, por ejemplo, que el argumento externo ensamblado en el especificador de Sv se interpreta como el Iniciador de un evento, tal como se propone en Jaque (2014: 156, cf. Ramchand 2008):

\section{(57) $\left\|v^{0}\right\|=\lambda P_{<e>} \lambda x \lambda e$.Iniciador(x, e) \& $\mathrm{P}(\mathrm{e})$}

Así, la denotación de $\mathrm{v}^{0}$ correspondería a una función que se aplica a propiedades de eventos (sintácticamente, SV) y arroja propiedades de individuos (x; sintácticamente, un SD) tales que el individuo en cuestión corresponde al iniciador de un evento P (v.g. bloquear, preocupar). En ausencia de $\mathrm{V}$, la derivación carece de variable eventiva $<\mathrm{e}>$, de forma tal que la función de (57) no puede aplicarse. En tales casos, sin embargo, diremos que el Sv es "defectivo", en el sentido de que su argumento externo no se relaciona con ningún evento, según comentaremos más adelante. La única implicación que busca explicitarse en esta formalización es la de que un argumento externo es estructuralmente causa solo si se ha ensamblado un evento en un paso anterior de la derivación. Es decir, no podemos definir dicho papel temático si no es con relación a un dominio espaciotemporal concreto en el que intervienen participantes (cf. Piñón 1997), representado en la derivación por $<\mathrm{e}>$. Esto no quiere decir, pues, que la causatividad como tal esté codificada en $\mathrm{v}$, que es un ingrediente necesario pero no suficiente. Se trata, en cambio, de una definición configuracional de la causatividad en 
la que ninguna proyección, en y por sí misma, codifica un valor causativo (cf. Cuervo 2015) ). $^{30}$.

De este modo, asignamos tanto a los verbos del tipo bloquear como del tipo preocupar la estructura siguiente:

(58) $\left[_{\mathrm{SV}} \mathrm{SD}_{\text {INCIADOR }} \mathrm{V}^{\circ}\left[\mathrm{SV}_{\mathrm{SV}} \mathrm{V}_{\text {OCP }}\left[{ }_{\mathrm{SRCC}} \mathrm{SD}_{\text {EXP/ARGINT }} \mathrm{R}_{\mathrm{CC}} \mathrm{SX}\right]\right]\right]$

En el caso de los verbos de alternancia instrumental (bloquear), la variación entre un valor estativo (v.g. la roca bloquea la entrada desde hace horas) y un valor eventivo dinámico (v.g. la roca está bloqueando poco a poco la entrada) puede computarse a partir del cambio de valor del núcleo $\mathrm{R}^{\circ}$, desde Coincidencia Central, para estado, a Coincidencia Terminal, para evento dinámico, siguiendo la pauta expuesta más arriba. En cambio, los verbos de experimentante objeto alternarían entre el valor aspectual codificado en (58) y una estructura estativa no causativa más simple, análoga a la de verbos de dativo léxico como gustar o doler, que carecería de proyección $\mathrm{V}$ y, por lo tanto, de argumento eventivo. De acuerdo con la definición de (57), una estructura carente de $<\mathrm{e}>$ no podría tomar un argumento externo marcado como Iniciador, toda vez que no habría un evento con el cual relacionar dicho papel temático. Este cambio aspectual se correlacionaría con las propiedades gramaticales revisadas en 3.4, es decir, admisibilidad de lecturas epistémicas y relación temporal $\mathrm{R} \subseteq \mathrm{E}$.

Para terminar, mencionaremos dos consecuencias del análisis arriba presentado, que vienen a reforzar su adecuación descriptiva para el español. En primer lugar, la división de la frase verbal en Sv, SV y SR abre la posibilidad teórica de que Sv se combine directamente con SR, sin ensamblar en medio SV. La predicción es que, en tal caso, no codificaríamos un valor genuinamente causativo, ya que, al faltar $<\mathrm{e}>$, tendríamos un estado puro y no un estado davidsoniano. Estimamos que dicha estructura se cumple

30 La propuesta de Cuervo es asimismo de tipo configuracional. Para la autora, el valor causativo emerge de la combinación de un $\mathrm{v}_{\mathrm{do}}$ con $\mathrm{v}_{\mathrm{be}}$, dando lugar a una representación bieventiva de los predicados causativos. $\mathrm{Si}$, en cambio, sobre $\mathrm{v}_{\mathrm{be}}$ se ensambla $\mathrm{v}_{\mathrm{go}}$, obtenemos un predicado inacusativo (complejo). La propuesta aquí presentada es similar en al menos dos puntos: (i) el valor causativo emerge de la combinación de proyecciones más básicas y (ii) la representación sintáctica de la causatividad es compleja (aunque no bieventiva, ya que en nuestra estructura se proyecta solo un argumento eventivo). Sin embargo, un punto importante en el que hemos querido ser aquí explícitos es en la independización de las nociones de causatividad y dinamicidad. En la propuesta de Cuervo (2015), al asociar la causatividad parcialmente con $\mathrm{v}_{\mathrm{do}}$, se la asocia con la dinamicidad, cuestión que, de acuerdo con los criterios empíricos esgrimidos, sería incorrecta, a menos que se clarifique el estatus aspectual del operador $d o$. 
en el caso del verbo estativo tener. Tal como se argumenta en Jaque (2014, en prensa), puede sostenerse que el verbo tener lexicaliza la proyección $\mathrm{v}$; consecuencias de ello serían la derivación en -dor (v.gr. Los tenedores de deuda soberana) y la asignación de caso acusativo (v.gr. María tiene la revista $>$ María la tiene). Ahora bien, un patrón altamente productivo en español es el de la combinación de tener con predicados secundarios de objeto directo, como se ejemplifica en (59):

(59) a. Tomás tiene su dinero en un paraíso fiscal.

b. Tomás tiene una mancha en el pantalón.

c. Tomás tiene el cabello castaño.

Considérese que tanto los SSPP de (59a-b) como el adjetivo de (59c) quedan fuera, como corresponde a complementos predicativos, de la pronominalización del objeto: Tomás \{lo tiene en un paraíso fiscal / la tiene en el pantalón / lo tiene castaño\}. Asumiendo la arquitectura verbal presentada, una forma de representar esta construcción corresponde a la combinación de Sv, cuyo núcleo lexicaliza tener, y SRcc, en el que se establece la relación predicativa del SD (especificador) y el SP o SA (complemento):

\section{(60) $\left[_{\mathrm{Sv}}\right.$ Tomás $\left[\mathrm{v}\right.$ tener $\left.\left.\left[{ }_{\mathrm{SRcc}} \mathrm{SD}\left[\mathrm{R}_{\mathrm{cc}} \mathrm{SP} / \mathrm{SA}\right]\right]\right]\right]$}

Ahora bien, si la estructura de (60) está bien encaminada, esperamos dos consecuencias. La primera es que, dado que no existe un evento, la función de (57) no puede aplicarse y no obtenemos, por tanto, un valor causativo. Esta predicción parece cumplirse, toda vez que, aunque podemos atribuir al sujeto de (59a) un valor conceptual de "responsabilidad", su carácter prescindible y no estructural se confirma al observar que, tanto en (59b) como $(59 \mathrm{c})$ no tiene ningún sentido conceptualizar al sujeto como iniciador de la circunstancia denotada por la predicación secundaria. En segundo lugar, el valor aspectual de dichas construcciones debe ser, crucialmente, de estado puro, no de estado davidsoniano. Al respecto, resulta particularmente claro el resultado que se obtiene al emplear contextos que admiten lectura epistémica orientada al presente: 
(61) a. Tomás tendrá su dinero en un paraíso fiscal (porque ningún fiscal ha podido encontrarlo).

b. Tomás debe tener una mancha en el pantalón (porque se tapa con la mano).

c. Tomás debe tener el cabello castaño (porque en la foto se le ve muy claro).

(62) a. Tomás obtendrá su dinero rápidamente.

b. Tomás debe contener las lágrimas en los ojos. (cf. ...debe estar conteniendo)

Como puede apreciarse, los usos de tener con predicados secundarios ofrecen una lectura epistémica tanto en futuro simple (61a) como bajo la perífrasis deber $+\inf (61 b-c)$. Esto contrasta con la conducta de derivados eventivos del mismo verbo: obtener (62a) o contener (62b), que imponen lecturas temporales o deónticas, respectivamente, a menos que se emplee primero una forma estativizadora (como la forma progresiva, en (62b)). Como es de esperar, en estos últimos casos sí emerge un valor causativo.

En segundo lugar, una pregunta que surge al considerar la estructura presentada en (58) es cómo habrían de modelarse otros tipos de eventos no contemplados en nuestro análisis, pero con los que la estructura propuesta, si ha de ser adecuada, debiese al menos ser compatible ${ }^{31}$. Por una parte, la representación de un evento dinámico no causativo se obtendría de la combinación de $\mathrm{V}$ con $\mathrm{R}_{\mathrm{c}}$, en ausencia de $\mathrm{v}$. Tal sería el caso de verbos inacusativos de cambio de estado, como hundirse u oscurecerse. Por otra parte, si los valores de $\mathrm{R}$ permiten modelar la alternancia entre evento no dinámico y evento dinámico télico, ¿cómo se representaría un evento dinámico atélico (correr, bailar, nadar)? En tal caso, seguimos la propuesta de Borer (2005), según la cual la atelicidad no corresponde, en rigor, a un valor aspectual sintácticamente codificado, sino al valor por defecto que un evento adopta en ausencia de otras especificaciones aspectuales; dicho de otro modo, lo que la sintaxis debe computar es si un evento es télico o estativo. En nuestros términos, si V no toma como complemento un SR, interpretaremos que, por defecto, se codifica un evento dinámico atélico, dado que no introducimos ni una condición de homogeneidad $\left(\mathrm{SR}_{\mathrm{CC}}\right)$ ni una de telicidad $\left(\mathrm{SR}_{\mathrm{cT}}\right)$. Algunas de las principales configuraciones admitidas, en relación con el tipo aspectual-argumental al que corresponden, se sintetizan en (63):

31 Debemos la consideración de esta cuestión a las observaciones de un revisor anónimo. 

(63) a. $\left[\mathrm{Sv}\left[\mathrm{SV}\left[\mathrm{SR}_{\mathrm{cc}}\right]\right]\right]$
b. $\left[\mathrm{Sv}\left[\mathrm{SV}\left[\mathrm{SR}_{\mathrm{CT}}\right]\right]\right]$
c. $\left[\mathrm{Sv}\left[\mathrm{SR}_{\mathrm{cc}}\right]\right]$
d. $\left[\mathrm{SV}\left[\mathrm{R}_{\mathrm{CT}}\right]\right]$
e. $[\mathrm{Sv}[\mathrm{SV}]]$

ESTADO DAVIDSONIANO CAUSATIVO

EVENTO DINÁMICO TÉLICO CAUSATIVO

ESTADO PURO CON PREDICACIÓN SECUNDARIA

EVENTO DINÁMICO INACUSATIVO

EVENTO ATÉLICO (DINÁMICO)

\section{CONCLUSIONES}

En este trabajo hemos analizado la categoría de estado causativo tal como se manifiesta en dos grupos de predicados del español: los verbos de alternancia instrumental (bloquear) y los VPEO de acusativo (preocupar). La generalización descriptiva principal que hemos sostenido es que, para ambos grupos de verbos, la categoría de estado SL resulta solo parcialmente adecuada, puesto que vuelve opaca la distinción entre estados SL y eventos no dinámicos o estados davisonianos. En concreto, decimos que los estados causativos pertenecen a la segunda de estas categorías, puesto que incluyen una variable eventiva $<$ e $>$ en su representación semántica.

Para comprobar esto, hemos visto cómo se comportan los predicados considerados ante dos contextos: la disponibilidad de lecturas epistémicas orientadas al presente y la relación de inclusión temporal entre E y R. El primer contexto diagnostica la evaluabilidad del predicado en puntos de tiempo o intervalos; la segunda muestra la presencia de un argumento eventivo, que impone una interpretación máxima a la localización temporal del estado de cosas. Hemos visto que ambos diagnósticos muestran de modo convergente que los estados causativos siguen el patrón de los estados davidsonianos, y no de los estados SL.

Finalmente, hemos propuesto que la teoría de sucesos de Moreno Cabrera (2003) da cuenta adecuadamente del carácter temporalmente extenso de los estados causativos, al restringir la combinación de un agente a procesos, sean de mantenimiento o de mutación, y bloquearla con estados puros. Desde un punto de vista sintáctico, hemos presentado un modelo en el que, siguiendo a Jaque $(2014,2016)$, la extensión temporal depende de la variable $<\mathrm{e}>-$ introducida por el núcleo $\mathrm{V}^{\mathrm{o}}$-, que proporciona validez en intervalos a la relación predicativa básica expresada en SR. Tal como sucede en el modelo de Moreno Cabrera, la obligada combinación de un argumento externo con eventos impide que los estados puros tengan valor causativo, garantizando así la distribución de las propiedades semánticas y gramaticales observadas. 


\section{REFERENCIAS BIBLIOGRÁFICAS}

Arad, Maya. 1999. What Counts as a Class? The Case of Psych Verbs. MIT Working Papers in Linguistics 35. Papers from the UPenn/MIT Roundtable on the Lexicon: 1-23.

Arche, María Jesús. 2006. Individuals in Time. Tense, aspect and the individual/stage distinction. Amsterdam/Philadelphia: John Benjamins.

Bach, EMmon. 1986. The Algebra of Events. Lingistics and Philosophy 9: 5-16.

BAKER, MARK. 1988. Incorporation: a theory of grammatical function changing. Chicago: University of Chicago Press.

Belletti, Adriana y Luigi Rizzi. 1988. Psych Verbs and Theta Theory. Natural Language and Linguistic Theory 6: 291-352.

Bennett, Michael y Bárbara Partee. 2004 [1978]. Toward the Logic of Tense and Aspect in English. En Barbara Partee (comp.). Compositionality in Formal Semantics. Selected Papers by Barbara H. Partee, pp. 59-109. Oxford: Blackwell.

Borer, Hagig. 2005. Structuring sense. Volume II. The Normal Course of Events. Oxford: Oxford UniversityPress.

Bosque, Ignacio. 1989. Las categorías gramaticales. Relaciones y diferencias. Madrid: Síntesis.

BRUCART, José. 2010. La alternancia ser/estar y las construcciones atributivas de localización. Actas del V Encuentro de gramática generativa, 29-31.07.2013, pp. 115-152. Universidad Nacional de Comahue, Río Negro, Argentina.

Bybee, Joan, Revere Perkins y William Pagliuca. 1994.The Evolution of Grammar. Tense, Aspect, and Modality in the Languages of the World. Chicago \& London: University of Chicago Press.

Carlson, Greg. 1977. Reference to Kinds in English. Tesis doctoral, University of California. 2012. Habitual and Generic Aspect. En Binnick, Robert I. Binnick (ed.). The Oxford Handbook of Tense and Aspect, pp. 828-851. Oxford University Press.

Сномsкy, Noam. 2001. Derivation by Phase. En Michael Kenstowicz (ed.). Ken Hale: A Life in Language, pp. 1-52. Cambridge: MIT Press.

Cifuentes Honrubia, José Luis. 2015. Causativity and psychological verbs in Spanish. En Elisa Barrajón, José Luis Cifuentes y Susana Rodríguez (eds.) Verb Classes and Aspect, pp. 110-130. Amsterdam: John Benjamins.

Cinque, Guglielmo. 1999. Adverbs and Functional Heads. Nueva York: Oxford University Press.

Cuervo, María Cristina. 2015. Causation without a Cause. Syntax 18(4): 388-424.

De Miguel, Elena. 1999. El aspecto léxico. En Ignacio Bosque y Violeta Demonte (dirs.). Gramática descriptiva de la lengua española, vol. 2, cap. 46. Madrid: Espasa.

Dowty, DAvid. 1979. Word Meaning and Montague Grammar. Dordrecht: Reidel.

FÁbregas, Antonio y Rafael Marín. 2015. Deriving individual-level and stage-level psych verbs. The Linguistic Review 32(2): 167-215.

2013. Entre estados y eventos: un análisis construccionista de las actividades no dinámicas. Ponencia presentada en el XLII Simposio de la Sociedad Española de Lingüística. Madrid, España.

GenNari, Silvia. 2002. Spanish past and future tenses: Less (semantics) is more. En Javier Gutérrez-Rexach (ed.). From words to discourse: Trends in Spanish semantics and pragmatics, pp. 21-36. Amsterdam: Elsevier.

Grimshaw, Jane. 1990. Argument Structure. Cambridge: MIT Press. 
Hale, Ken. 1984. Notes on world view and semantic categories: Some Warlpiri examples. En Pieter Muysken \& Henk van Riemsdijk (eds.). Features and Projections, pp. 233-254. Dordrecht: Foris.

Hale, Ken y Samuel J. Kayser. 2002. Prolegomenon to a Theory of Argument Structure. Cambridge: The MIT Press.

Hallman, Peter. 2009. Instants and intervals in the event/state distinction. Manuscrito no publicado disponible en http://peterhallman.com/States.pdf.[Consulta: 15.06.13].

Harley, Heidi. 2009. The morphology of nominalizations and the syntax of vP. En Anastasia Giannakidou y Monika Rathert (eds.) Quantification, Definiteness, \& Nominalization, pp. 321-343. Oxford: Oxford University Press.

HusBand, E. MatThew. 2010. On the compositional nature of stativity. Tesis doctoral, Michigan State University.

JAQUE, MATÍAS. 2014. La expresión de la estatividad en español: niveles de representación y grados de dinamicidad. Tesis doctoral, Universidad Autónoma de Madrid. 2016. Relaciones entre aspecto y modalidad epistémica: algunas consecuencias de las restricciones temporales sobre la evaluación de predicados. Onomazein 33: 130-155. (en prensa). Niveles de estatividad en el Sv: algunas consecuencias gramaticales en español. Revista de la Sociedad Española de Lingüística.

Katz, Graham. 2000. Anti neo-Davidsonianism: against a Davidsonian semantics for state sentences. En James Pustejovsky y Carol Tenny (eds.). Events as Grammatical Objects. The converging perspectives of lexical semantics and syntax, pp. 393-416. Standford: CSLI.

Klein, Wolfgang. 1994. Time in Language. London: Routledge.

Kratzer, Angelika. 1995. Stage-level and Individual level predicates. En Greg Carlson y Francis Pelletier (eds.). The Generic Book, pp. 125-175. Chicago IL: Chicago University Press.

1996. Severing the External Argument from its Verb. En Johan Rooryck \& Laurie Zaring (eds.). Phrase Structure and the Lexicon, pp. 109-137. Dordrecht: Kluwer.

Landau, Idan. 2009. The Locative Syntax of Experiencers. Cambridge, MA: MIT Press.

LEWIS, DAviD. 1973. Counterfactuals. Oxford: Blackwell.

1973b. Causation. The Journal of Philosophy 70(17): 556-567.

LUNDQUIST, BJöRN. 2012. Localizing cross-linguistic variation in Tense systems: on telicity and stativity in Swedish and English. Nordic Journal of Linguistics 35(1): 27-70.

MaienBorn, Claudia. 2005. On the limits of The Davidsonian approach: The case of copula sentences. Theoretical Linguistics 31: 275-316.

Marín, Rafael. 2011. Casi todos los predicados psicológicos son estativos. En Ángeles Carrasco (ed.). Sobre estados y estatividad, pp. 26-44. Muenchen: LINCOM.

2013. La stativité dans tous ses états, Memoria de habilitación, Université Paris 8.

2015. Los predicados psicológicos. Debate sobre el estado de la cuestión. En Rafael Marín (ed.). Los predicados psicológicos, pp. 11-50. Madrid: Visor.

Marín, Rafael y Louise McNally. 2011. Inchoativity, change of state, and telicity: Evidence from Spanish reflexive psychological verbs. Natural Language and Linguistic Theory 48(1): 35-70.

Mateu, Jaume. 2002. Argument Structure. Relational Construal at the Syntax-Semantics Interface. Tesis doctoral. Universitat Autònoma de Barcelona, Bellaterra.

Mitтwoch, Anita. 1988. Aspects of English Aspect: on the Interaction of Perfect, Progressive and Durational Phrases. Linguistics and Philosophy 11: 203-254.

Moreno Cabrera, Juan Carlos. 2003. Semántica y gramática. Madrid: Machado Libros. 
Neeleman, Ad y Hans van de Koot. 2012. The Linguistic Expression of Causation. En Martin Everaert, Marijana Marelj y Tal Siloni (eds.). The Theta System, pp. 279-307. Oxford: Oxford University Press.

Parsons, Terence. 1989. The Progressive in English: Events, States and Processes. Linguistics and Philosophy 12: 213-241.

Pesetsky, David.1995. Zero Syntax. Cambridge, MA: MIT Press.

Piñón, Christopher. 1997. Achievements in an Event Semantics. Proceedings of SALT VII, pp. 276-293. Ithaca, NY, Cornell University.

Portner, Paul. 2005. What is meaning? Fundamentals of Formal Semantics. Oxford: Blackwell.

PylkKänen, LiIna. 2000. On Stativity and Causation.En C. Tenny and J. Pustejovsky (eds.). Events as Grammatical Objects, pp. 417-442. Stanford, CSLI. 2002. Introducing Arguments. Tesis doctoral. MIT, Boston.

Ramchand, Gillian. 2008. Verb Meaning and the Lexicon. Cambridge: Cambridge University Press.

Reichenbach, Hans. 1947. Elements of symbolic logic. London: Macmillan.

Rothmayr, Antonia. 2009. The Structure of Stative Verbs. Amsterdam: John Benjamins.

Soto, Guillermo. 2008. Sobre el llamado futuro de probabilidad. Algunas condiciones del valor modal de -ré. Boletín de Filología XLIII: 193-206.

Strozer, J. R. 1976. Clitics in Spanish. Tesis doctoral, UCLA.

TAlmy, LeONARD. 1972. Semantic structures in English and Atsugewi. Tesis doctoral, Department of Linguistics, University of California, Berkeley.

TAYLOR, BARry. 1977. Tense and Continuity. Linguistics and Philosophy 1.2: 199-220.

Tenny, Carol. 1994. Aspectual roles and the Syntax-Semantics Interface. Dordrecht: Kluwer. Verkuyl, Henk. 1993. A Theory of Aspectuality. Cambridge: Cambridge UniversityPress.

Vlach, Frank. 1981. The Semantics of the Progressive. En Philip Tedeschi y Annie Zaenen (eds.). Syntax and Semantics 14: Tense and Aspect, pp. 271-292. Nueva York: Academic Press. 